Kennesaw State University

DigitalCommons@Kennesaw State University

Faculty Publications

4-1996

\title{
Stochastic Electron Acceleration by Cascading Fast Mode Waves in Impulsive Solar Flares
}

James A. Miller

University of Alabama - Tuscaloosa

Ted N. La Rosa

Kennesaw State University, tlarosa1@kennesaw.edu

Ronald L. Moore

NASA

Follow this and additional works at: https://digitalcommons.kennesaw.edu/facpubs

Part of the Stars, Interstellar Medium and the Galaxy Commons, and the The Sun and the Solar System Commons

\section{Recommended Citation}

Miller JA, LaRosa TN, Moore RL. 1996. Stochastic electron acceleration by cascading fast mode waves in impulsive solar flares. Astrophys J 461(1):445-64.

This Article is brought to you for free and open access by DigitalCommons@Kennesaw State University. It has been accepted for inclusion in Faculty Publications by an authorized administrator of DigitalCommons@Kennesaw State University. For more information, please contact 
The AstrophysiCAL Journal, 461:445-464, 1996 April 10

(C) 1996. The American Astronomical Society. All rights reserved. Printed in U.S.A.

\title{
STOCHASTIC ELECTRON ACCELERATION BY CASCADING FAST MODE WAVES IN IMPULSIVE SOLAR FLARES
}

\author{
JAMES A. MiLLER \\ Department of Physics, University of Alabama in Huntsville, Huntsville, AL 35899; miller@mpingo.uah.edu \\ T. N. LARosa \\ Department of Biological and Physical Sciences, Kennesaw State College, P.O. Box 444, Marietta, GA 30061 \\ AND \\ R. L. MOORE \\ Space Sciences Laboratory, NASA/MSFC, Huntsville, AL 35812 \\ Received 1995 August 8; accepted 1995 October 13
}

\begin{abstract}
We present a model for the acceleration of electrons from thermal to ultrarelativistic energies during an energy release fragment in an impulsive solar flare. Long-wavelength low-amplitude fast mode waves are assumed to be generated during the initial flare energy release (by, for example, large-scale restructuring of the magnetic field). These waves nonlinearly cascade to higher wavenumbers and eventually reach the dissipation range, whereupon they are transit-time damped by electrons in the tail of the thermal distribution. The electrons, in turn, are energized out of the tail and into substantially higher energies. We find that for turbulence energy densities much smaller than the ambient magnetic field energy density and comparable to the thermal particle energy density, and for a wide range of initial wavelengths, a sufficient number of electrons are accelerated to hard X-ray-producing energies on observed timescales. We suggest that MHD turbulence unifies electron and proton acceleration in impulsive solar flares, since a preceding study established that a second MHD mode (the shear Alfvén wave) preferentially accelerates protons from thermal to gamma-ray line-producing energies.

Subject headings: acceleration of particles - plasmas - Sun: corona - Sun: flares -

Sun: particle emission
\end{abstract}

\section{INTRODUCTION}

The acceleration of up to $10^{38}$ electrons to energies above $20 \mathrm{keV}$ on timescales of the order of $100 \mathrm{~s}$ or less (e.g., Hoyng, Brown, \& van Beek 1976) has been one of the outstanding problems in high-energy solar flare physics and, along with the associated problem of ion acceleration and abundance enhancements (see Reames, Meyer, \& von Rosenvinge 1994 and references therein), is at the heart of solar flare research. While the duration of the entire impulsive phase can last for this length of time, there is considerable evidence that some flares exhibit a much shorter timescale for hard X-ray production and concomitant electron acceleration. Data obtained with the Hard X-Ray Burst Spectrometer on the Solar Maximum Mission have shown spikes of duration as small as $\approx 400 \mathrm{~ms}$ superposed upon the more slowly varying background of hard X-rays (Kiplinger et al. 1984). Employing a thick-target model for the hard X-ray production (e.g., Brown 1971; Emslie 1983), Kiplinger et al. deduce that about $2 \times 10^{34}$ electrons were accelerated to energies greater than $20 \mathrm{keV}$ in one of these spikes. With the aforementioned spike duration, the rate at which electrons are energized above $20 \mathrm{keV}$ is then $\approx 5 \times 10^{34} \mathrm{~s}^{-1}$. Subsequent confirmation that energy release and electron acceleration can be episodic or fragmented was provided by decimetric radio emission (e.g., Benz 1985; Güdel, Aschwanden, \& Benz 1991).

More recently, the existence of a subsecond spiky structure in hard X-ray emission from some impulsive flares has been confirmed by observations made with the Burst and Transient Spectrometer on the Compton Gamma Ray Observatory (Machado et al. 1993; see also Aschwanden,
Schwartz, \& Alt 1995) and has prompted Machado et al. to call these spikes "energy release fragments" and hypothesize that they are the basic constituent of the entire impulsive phase. The energy content of hard X-ray-producing electrons in a fragment is between $10^{26}$ and $10^{27}$ ergs, since the number of electrons that were accelerated to energies above $\approx 20 \mathrm{keV}$ lies in the range from $\approx 3 \times 10^{33}$ to $\approx 3 \times 10^{34}$. For a duration of $\approx 300 \mathrm{~ms}$, the rate of energization above $20 \mathrm{keV}$ in a fragment is then $\approx 10^{34}-\approx 10^{35}$ $\mathrm{s}^{-1}$. Hence, in light of the hard X-ray observations, a general requirement of any electron acceleration process is that it be able to energize $\approx 5 \times 10^{34}$ electrons $\mathrm{s}^{-1}$ above 20 $\mathrm{keV}$ over $\approx 400 \mathrm{~ms}$ and thus yield about $2 \times 10^{34}$ electrons above this energy. We consider energy release fragments in this paper and address only this basic observation. Of course, another very important test of any mechanism is its ability to produce electron energy distributions consistent with hard X-ray spectra. However, for the present, we consider this a secondary issue, linked to transport, and to be explored once the self-consistent and fully time-dependent behavior of an acceleration model is established.

It is not known whether all impulsive solar flares are indeed composed of a large number of these basic elements or fragments. If this is so, then by considering the elementary event, we have essentially solved the electron acceleration problem for the entire duration of the hard X-ray emission. If not, and the acceleration is more steady and less punctuated in some instances, our stochastic mechanism will still work but requires an extended injection of turbulence. In either case, the acceleration region must be repopulated for about the duration of the energization due 
to the very large number of electrons that need to be accelerated and need to interact in the footpoints of the loops. This replenishment can be accomplished by a cospatial return current from the electron-rich partially ionized chromosphere, which is readily established, either inductively (Spicer \& Sudan 1984) or electrostatically (LaRosa \& Emslie 1989), or both (van den Oord 1990), in response to the drift of the accelerated electrons to the loop footpoints. As opposed to large-scale electric field acceleration (Benka \& Holman 1994), stochastic acceleration mechanisms do not require filamentation in the form of $10^{6}-10^{12}$ oppositely directed current/return-current pairs (Emslie \& Hénoux 1995) and, in fact, may need none at all. While Emslie \& Hénoux showed that current closure in such a fine-scale environment may be accomplished, the source of the initial filamentation is unknown. A detailed treatment of extended acceleration and cospatial return currents (e.g., Emslie 1980) is beyond the scope of this paper but will be presented in the future.

We propose that electrons are accelerated from thermal to relativistic energies by resonance with low-amplitude fast mode waves in a continuous broad-band spectrum. The fast mode branch in a plasma extends from low frequencies, past the hydrogen cyclotron frequency $\Omega_{\mathrm{H}}$, and up to the electron cyclotron frequency (see, e.g., Swanson 1989, chap. 2). Fast mode waves on the $\omega \ll \Omega_{H}$ section of this branch have a dispersion relation given by $\omega=v_{\mathrm{A}} k$, where $v_{\mathrm{A}}, k$, and $\omega$ are the Alfven speed, the magnitude of the wavevector $\boldsymbol{k}$, and the wave frequency, respectively. As $\omega$ approaches $\Omega_{\mathrm{H}}$, this simple relation is no longer valid, and above $\approx 10 \Omega_{\mathrm{H}}$ the branch enters the whistler regime. The waves that are important for electron acceleration in our theory have an average frequency less than $\approx 0.14 \Omega_{\mathrm{H}}$ and lie below the whistler regime. Low-frequency (MHD) fast mode waves have a simple polarization. If a wave is parallel, then its electric field $\boldsymbol{E}_{\boldsymbol{w}}$ is transverse to the ambient magnetic field $\boldsymbol{B}_{0}$ and right-hand circularly polarized; if it is oblique, then the wave is linearly polarized, and $\boldsymbol{E}_{\boldsymbol{w}}$ is in the direction of $\boldsymbol{B}_{0} \times \boldsymbol{k}$. From Faraday's Law, then, the wave magnetic field $\boldsymbol{B}_{w}$ has both a compressive (along $\boldsymbol{B}_{0}$ ) and a linearly polarized transverse component.

The wave electric field can strongly affect particle motion through gyroresonant interactions (e.g., Karimabadi, Omidi, \& Gary 1994 and references therein), the condition for which is $\omega-k_{\|} v_{\|}-l \Omega / \gamma=0$, where $v_{\|}=\mu v, \gamma$, and $\Omega=|q| B_{0} / m c$ are the parallel speed, Lorentz factor, and gyrofrequency of the particle, and $k_{\|}=\eta k$ is the parallel wavenumber. For parallel propagation, the low-frequency fast mode wave electric field can gyroresonate with only relativistic electrons via $l=+1$ (Steinacker \& Miller 1992) or relativistic ions via $l=-1$. In the oblique case, $\boldsymbol{E}_{w}$ can be decomposed into right- and left-hand circularly polarized components, and the left-hand portion can only resonate with relativistic ions via $l=+1$ or relativistic electrons via $l=-1$ (Steinacker $\&$ Miller 1992). Therefore, the low-frequency wave electric field is relevant for only relativistic particles and is of no use in accelerating electhermal background.

However, the compressive component of $\boldsymbol{B}_{w}$ can also interact with particles, but through the $l=0$ resonance. This process is called transit-time damping [Fisk 1976; Stix 1992 , p. 273 (this terminology is also present in the 1962 edition of his text)] and is the magnetic analog of Landau damping (which involves the $l=0$ resonance and a parallel electric field). In transit-time damping, the interaction is between the magnetic moment of a particle and the parallel gradient of the magnetic field. Using the low-frequency fast mode wave dispersion relation, the condition for resonance to occur is $v_{\|}=v_{\mathrm{A}} / \eta$ and implies that the particle and wave always move in the same direction (as opposed to gyroresonant interactions). From this condition we see that the threshold speed, below which resonance is not possible for any wave $\eta$ or pitch-angle cosine $\mu$, is the Alfvén speed $v_{\mathrm{A}}$. For speeds $v>v_{\mathrm{A}}$, we can readily determine the efficiency of the interaction for an isotropic particle distribution. Specifically, if there are waves with $\eta$ in the interval $\left[+v_{\mathrm{A}} / v,+1\right]$ and in the interval $\left[-1,-v_{\mathrm{A}} / v\right]$, then $100 \%\left[1-\left(v_{\mathrm{A}} / v\right)\right]$ of the particles will be able to resonate with the waves. Hence, for $v \approx v_{\mathrm{A}}$, only a small fraction of the particles will be able to interact with waves, which propagate nearly parallel or antiparallel to $\boldsymbol{B}_{0}$. However, this fraction is nearly $100 \%$ if $v \approx c$, and the resonant waves have broad distribution in propagation angle. Note that waves with $\eta$ between $-v_{\mathrm{A}} / c$ and $+v_{\mathrm{A}} / c$ will not be able to resonate with particles of any energy.

The essential point here is that the most efficient acceleration from thermal to relativistic energies is achieved when there is a distribution of waves between $\eta=+v_{\mathrm{A}} / c$ and +1 and between $-v_{\mathrm{A}} / c$ and -1 . Physically, resonance with one wave results in an energy change that brings the particle into resonance with a neighboring wave, which then changes the energy so as to allow the particle to resonate with another wave, and so on (see Karimabadi, KraussVarban, \& Terasawa 1992). The energy change is diffusive, but over long timescales there is a net gain of energy, resulting in stochastic acceleration.

This process is essentially the resonant form of Fermi acceleration (Fermi 1949; Davis 1956). Here, a particle's magnetic moment is repelled upon encountering a region of sufficiently high increased flux density. If the gyroradius is much smaller than the scale of the perturbation, the first adiabatic invariant is conserved in a collision. Particles can make either head-on or trailing collisions with a moving fluctuation: If the collision is head on (trailing), the particle will be reflected with an increase (decrease) in energy. Head-on collisions occur more often per unit time because of the higher relative velocity between the particle and the perturbation, and there will hence be a net acceleration. This picture is strictly correct only when the strength of the compressions is high enough so that most particles can be reflected before completely penetrating the region of the compression, and they could correspond to large-amplitude fast mode waves. As the amplitude decreases, the number of particles that are reflected decreases. In the limit of very small amplitudes, the parallel particle speed in the wave frame moving along $\boldsymbol{B}_{0}$ with the parallel phase speed must be about zero for reflection to occur. That is, $v_{\|}=\omega / k_{\|}$, which is just the above resonance condition. Hence, the process we are considering could be called small-amplitude Fermi acceleration (Achterberg 1981); however, in keeping with earlier terminology, we call it acceleration by transittime damping, which clearly denotes the resonant character of the interaction.

An important aspect of transit-time acceleration is isotropization, since the $l=0$ resonance changes only the parallel energy of the particle. In the absence of ancillary pitch-angle scattering, transit-time acceleration would lead to a systematic decrease of particle pitch angles. Acceler- 
ation would then become less efficient, since only those waves with very high parallel phase speeds (i.e., those propagating at angles close to $90^{\circ}$ with respect to $\boldsymbol{B}_{0}$ ) would be able to resonate with the particles. For example, suppose that the parallel and perpendicular energy of an electron were both $\approx 0.1 \mathrm{keV}$ initially, which is the case for a thermal plasma of temperature $\sim 10^{6} \mathrm{~K}$. In order to account for solar flare hard X-ray observations, electrons must be accelerated up to at least $\approx 100 \mathrm{keV}$. In the absence of pitchangle scattering, this energy must all be in the parallel direction, in which case $v_{\|} \approx 0.55 c$ and $\mu \approx 0.999$. For an Alfvén speed of $0.036 c$ (see below), the resonance condition implies that $\eta$ be about $6.5 \times 10^{-2}$. Hence, in order to resonate with such an energetic electron, the waves need to propagate at about $86^{\circ}$ with respect to $\boldsymbol{B}_{0}$. On the other hand, if the electrons are isotropic, the pitch-angle cosine $\mu$ (and thus $v_{\|}$) would not be constrained to one value, and waves over a broad range of propagation angle would be able to interact with the electrons and contribute to their energization. Essentially, therefore, pitch-angle scattering greatly increases the volume of wave phase space that can be sampled by the particles, thus increasing the acceleration efficiency. We do not address the details of isotropization in this paper but rather assume that it exists and keeps the distribution isotropic during transit-time acceleration. Possible efficient scattering mechanisms include gyroresonant interactions with whistlers (e.g., Steinacker \& Miller 1992) that may also exist in the flare environment or gyroresonant interactions with lower hybrid waves generated by the accelerated electrons themselves (see $\S 2$ ).

The threshold speed of the resonance determines the selectivity of the process. In a solar flare hydrogen plasma with a temperature $T$ of $3 \times 10^{6} \mathrm{~K}$, density $n_{\mathrm{H}}$ of $10^{10}$ $\mathrm{cm}^{-3}$, and $B_{0}$ of $500 \mathrm{G}$, the electron thermal speed $v_{\mathrm{te}}=$ $0.032 c$, the proton thermal speed is $7.4 \times 10^{-4} c$, and the Alfvén speed is $0.036 c$. Therefore, the threshold speed is far in the tail of the proton distribution, and a negligible number of protons will be accelerated. Consequently, protons (and other ions) are a negligible dissipation source for the waves. On the other hand, $v_{\mathrm{A}}$ is only slightly above $v_{\text {te }}$, and a significant number of the ambient electrons can resonate with, and damp, the waves. Thus, fast mode waves almost exclusively accelerate electrons under solar flare conditions, although this situation may change in other environments (e.g., Fisk 1976).

In the classical Fermi process, the acceleration rate is proportional to the collision frequency between a particle and the magnetic perturbations. Identifying the collision frequency with the inverse of the transit time across a perturbation, and taking the perturbation to be a largeamplitude wave, we see that the acceleration rate increases as the wavenumber increases. This should be the case for transit-time damping as well, due to the analogy between the two processes. Qualitatively then, fast mode waves of relatively high $k$ will probably be necessary to accelerate electrons out of the background on short timescales and against Coulomb collisions. The generation of turbulence in flares is fairly unexplored territory, but it is reasonable to suppose that very long wavelength waves (of order $10^{8} \mathrm{~cm}$, say) will be excited during the primary energy release, by large-scale magnetic field restructuring or perhaps a shear flow instability (Chiueh \& Zweibel 1987; LaRosa \& Moore 1993; see also Roberts et al. 1992). The transfer of spectral energy to higher wavenumbers is naturally accomplished by cascading (e.g., Zhou \& Matthaeus 1990; Marsch 1991, $\$ \S 8.6$ and 10.5), which results physically from wave steepening.

In our scenario then, waves are initially excited at very long wavelengths, cascade through an inertial range, eventually reach a sufficiently high $k$ where they preferentially accelerate electrons out of the thermal distribution, and are subsequently damped. The range of $k$ where damping is significant is the dissipation range, the location of which depends upon the wave energy absorption rate due to transit-time damping relative to the wave energy cascade rate. We employ small-amplitude waves since we wish to consider the sufficient conditions under which the observed acceleration can take place and thus minimize the energy density of the turbulence. Since the energy density of the turbulence will be small and only those electrons above the threshold will be energized, the volume of the acceleration region will need to be relatively large $\left(\sim 10^{27} \mathrm{~cm}^{3}\right)$ to account for the total energy content of the electrons. An opposite scenario, in which the waves have large amplitudes and the volume of the acceleration region is only $\sim 10^{24}$ $\mathrm{cm}^{3}$, is considered in LaRosa, Moore, \& Shore (1994). In this case, electrons are accelerated by the Fermi mechanism and there is no threshold energy, so that the result is "bulk energization" of the entire distribution to hard X-rayproducing energies. An important point with transit-time damping (which is also the case with the associated Fermi mechanism) is that there is no often-quoted "injection problem:" A preacceleration mechanism is not needed, and this single process can accelerate electrons to ultrarelativistic energies directly from the thermal distribution.

Cascading was also an essential ingredient in the Fermi acceleration model of LaRosa et al. (1994). That Fermi acceleration of electrons could occur and be efficient was previously established by Gisler \& Lemons (1990) and Gisler (1992), studies which firmly refuted Eichler's (1979) claim that this process would always lead to heating. Acceleration by transit-time damping has been treated in the literature before. Achterberg (1981; see also 1979) derived approximate diffusion coefficients and also showed that the process can lead to acceleration. Miller (1991) showed that the dominant dissipation mechanism for fast mode waves under solar flare conditions was electron transit-time damping, and that the damping rate (and thus the electron acceleration rate) was very high. The study which cast doubt on both resonant and Fermi acceleration by fast mode waves was Zweibel \& de la Beaujardière (1990; see also de la Beaujardière \& Zweibel 1989), in which testparticle simulations revealed that the acceleration rate was too slow to account for electron energization in flares. We suspect the cause of this was the use of waves at low $k$. We also employ low- $k$ waves but take into account the subsequent transfer of spectral energy to smaller scales, where it is more rapidly dissipated by electron acceleration.

Herein, we formulate a self-consistent model for electron acceleration, taking into account the cascading of the waves, their damping on the electrons, and the resulting energization of the electrons. We pay careful attention to Coulomb collisions, since the threshold energy is near the thermal speed. Cascading and acceleration are treated with coupled diffusion equations, one of which is nonlinear. These equations are numerically solved by finite differencing with iteration. We discuss the model in detail in $\S 2$. In $\S 3$ we present results from the quasilinear code. Implica- 
tions for flare acceleration are discussed in $\S 4$, and we summarize our findings in $\S 5$. A derivation of the transittime damping diffusion coefficient, without approximation, is given in the Appendix and will be used here and in future extensions of this model.

\section{THE MODEL}

We assume that the acceleration region consists of an isotropic fully ionized $\mathrm{H}$ plasma permeated by a homogeneous static ambient magnetic field $\boldsymbol{B}_{0}=B_{0} \hat{z}$. At some large-scale $\lambda_{i}$, an unspecified mechanism generates smallamplitude fast mode waves, which then cascade to smaller scales and eventually accelerate electrons out of the thermal distribution. In the next two subsections, we present the quasilinear equations that describe the behavior of the electrons and the waves. Here, we summarize our assumptions and simplifications.

1. The turbulence is isotropic, which is consistent with the Kolmogorov-like and Kraichnan cascading phenomenologies that we employ. In the Kolmogorov-like phenomenology, waves of comparable wavelength interact in about one eddy turnover time, which is then the timescale for spectral energy transfer to smaller wavelength fluctuations. In the Kraichnan phenomenology, the interaction timescale is the Alfvén crossing time for a fluctuation. For lowamplitude waves, this interaction is much shorter than that for the Kolmogorov case, and spectral energy transfer to smaller scales is inhibited and occurs at a slower rate.

2. The electron distribution is isotropic. While this is likely to be the case initially, transit-time damping changes only the parallel energy of the particles. In the absence of pitch-angle scattering, the parallel energy would systematically increase, leading to a velocity-space anisotropy in the electron distribution function. This would decrease the efficiency of the acceleration by reducing the wavenumber phase space accessible to the energetic particles. However, as a tail is formed in the parallel direction, the anomalous Doppler resonance $(l=-1)$ instability (Liu \& Mok 1977; An et al. 1982; Moghaddam-Taaheri et al. 1985) would rapidly lead to the excitation of electrostatic lower hybrid waves that would pitch-angle scatter the particles back to a nearly isotropic state. This process needs to be investigated further in our context, but we assume it is operable over an acceleration timescale. We point out, however, that transittime damping may be efficient enough even without isotropizing scattering; this is to be investigated in the future.

3. Gyroresonance between the relativistic electrons and the transverse wave electric field (see $\S 1$ ) is neglected. This process will greatly enhance the acceleration rate of relativistic electrons over that obtained from just the $l=0$ interaction.

4. Electron escape from the acceleration region is not considered. Escape is dictated by the mechanism responsible for isotropization (see point 2), and, until this process is explored seif-consistently, we prefer not to include arbitrary parameters for it. (Note that this is not a problem in Alvén wave/ion acceleration models where the isotropizing and energizing waves are the same.) While not a critical simplification in this paper, since we are concerned with the overall efficiency of transit-time damping and its ability to produce the observed electron numbers, a treatment of escape is essential for a calculation of hard X-ray spectra.
5. We use the transit-time damping diffusion coefficient that results for waves with $\omega \ll \Omega_{\mathrm{H}}$. With Kraichnan cascading, the waves are confined to low frequencies and this simplification will have no effect. With Kolmogorov-like cascading, waves can have $\omega \approx \Omega_{\mathrm{H}}$, but the effect of the more complicated dispersion in this regime will not be significant.

\subsection{The Electron Diffusion Equation}

With the introduction of isotropizing scattering, there is no evolution of the particle distribution function in pitchangle cosine $\mu$. Hence, we can average the two-dimensional momentum diffusion equation in spherical coordinates over $\mu$ and readily obtain the isotropic momentum diffusion equation

$$
\frac{\partial f}{\partial t}=\frac{1}{p^{2}} \frac{\partial}{\partial p}\left[p^{2} D(p) \frac{\partial f}{\partial p}\right]
$$

where

$$
D(p)=\frac{1}{2} \int_{-1}^{+1} d \mu D_{p p},
$$

$p$ is the magnitude of the momentum vector $p$, and $D_{p p}=$ $\langle\Delta p \Delta p\rangle /(2 \Delta t)$ is the $\mu$-dependent momentum diffusion coefficient. The quantity $f$ is the phase-space distribution function, normalized such that $4 \pi p^{2} d p f(p, t)$ equals the number of particles per unit volume with momentum in the interval $d p$ about $p$. The transit-time damping diffusion coefficient $D_{p p}$ is derived in the Appendix using a convenient Hamiltonian formalism (e.g., Karimabadi et al. 1992; Miller \& Roberts 1995). From equations (2.1b) and (A10), we find that

$D(p)=(m c)^{2} \Omega_{\mathrm{H}} \frac{\pi}{16}\left(\frac{v_{\mathrm{A}}}{c}\right)^{2} \frac{c\langle k\rangle}{\Omega_{\mathrm{H}}} \frac{U_{\mathrm{T}}}{U_{\mathrm{B}}}\left(\frac{p}{m c}\right)^{2}\left(\frac{c}{v}\right) F(v)$,

where

$$
F(v)=-\frac{5}{4}-\left(1+2 \mu_{0}^{2}\right) \ln \mu_{0}+\mu_{0}^{2}+\frac{1}{4} \mu_{0}^{4},
$$

and $\mu_{0}=v_{\mathrm{A}} / v$. An electron of speed $v>v_{\mathrm{A}}$ must have $|\mu|>$ $\mu_{0}$ in order to resonate with a wave. In this expression, $m$ is the electron mass, $U_{T}$ is the total (kinetic plus vacuum field) energy density in the fast mode waves, $U_{B}=B_{0}^{2} / 8 \pi$ is the background magnetic field energy density, and $c\langle k\rangle / \Omega_{\mathrm{H}}$ is the mean dimensionless wavenumber of the wave spectrum. The quantity $F(v)$ is basically an efficiency factor, which takes into account the velocity-dependent fraction of particles that can resonate with waves having an isotropic distribution, and equals 0 when $v \leq v_{\mathrm{A}}$ but approaches $-(5 / 4)-\ln \left(v_{\mathrm{A}} / c\right) \approx 2$ when the electron becomes ultrarelativistic. This expression for $D(p)$ is equal to $3 F(v)$ times the expression derived by Achterberg (1981; see also 1979 and Forman, Ramaty, \& Zweibel 1986). Since $F(v) \lesssim 10^{-3}$ for $E \lesssim 10^{-3} m c^{2}$, the efficiency of low-energy electron acceleration is much smaller than previously estimated; however, the diffusion coefficient, and thus the acceleration rate, at high energies is larger by a factor of $\approx 6$.

Instead of the momentum diffusion equation, we finite difference the associated Fokker-Planck equation in energy space (Tsytovich 1966), which reads

$$
\frac{\partial N}{\partial t}=-\frac{\partial}{\partial E}\left\{\left[A+\left(\frac{d E}{d t}\right)_{\mathrm{C}}\right] N\right\}+\frac{1}{2} \frac{\partial^{2}}{\partial E^{2}}\left[\left(D+D_{\mathrm{C}}\right) N\right],
$$


where $E$ is kinetic energy and $N$ is the energy-differential particle density. The coefficient of systematic acceleration (or convection coefficient) $A(E)=\langle\Delta E\rangle / \Delta t$, which is given in terms of $D(p)$ by $p^{-2} \partial\left[p^{2} v D(p)\right] / \partial p$. The diffusion coefficient $D(E)=\left\langle(\Delta E)^{2}\right\rangle / \Delta t$ and can be written as $2 v^{2} D(p)$. We have also added a convection $(d E / d t)_{\mathrm{C}}$ and diffusion coefficient $D_{\mathrm{C}}(E)$ for Coulomb collisions. We treat the FokkerPlanck equation instead of the momentum diffusion equation since (1) the boundary condition at high energies is more easily implemented, (2) $A(E)$ is necessary for the calculation of the damping rate anyway (see $\S 2.2$ ), and (3) the energy convection and diffusion coefficients for Coulomb collisions have already been calculated. Differentiating $D(p)$, find that

$$
A(E)=\left(m c^{2}\right) \Omega_{\mathrm{H}} \frac{\pi}{4}\left(\frac{v_{\mathrm{A}}}{c}\right)^{2} \frac{U_{\mathrm{T}}}{U_{\mathrm{B}}} \frac{c\langle k\rangle}{\Omega_{\mathrm{H}}}\left(\frac{p}{m c}\right) \mathrm{G}(v),
$$

where

$$
\mathrm{G}(v)=\frac{1}{4 \gamma^{2}}\left(4 \mu_{0}^{2} \ln \mu_{0}-\mu_{0}^{4}+1\right)+F(p),
$$

and $\gamma$ is the Lorentz factor. The quantity $\mathrm{G}(v)=0$ when $v \leq v_{\mathrm{A}}$ and reaches a high energy limit of $\approx 1 /\left(4 \gamma^{2}\right)-(5 / 4)$ $-\ln \left(v_{\mathrm{A}} / c\right) \approx 2$. Note that the acceleration rate is directly proportional to $\langle k\rangle$, so that waves of relatively small wavelength are much more efficient energizers than those with large wavelength, in agreement with the argument presented in $\S 1$.

For all but the very lowest energies $\left(E \lesssim 10^{-2} k_{\mathrm{B}} T\right.$, where $k_{\mathrm{B}}$ is Boltzmann's constant and $T$ is the temperature), the energy change rate due to Coulomb collisions on the background electrons dominates that due to the background protons. Consequently, we consider only electron-electron collisions. In a fully ionized thermal $\mathrm{H}$ plasma, the Coulomb convection coefficient from Huba (1994) is

$$
\left(\frac{d E}{d t}\right)_{\mathrm{C}}=-2 v_{0}\left[\psi(x)-\psi^{\prime}(x)\right] E,
$$

where the diffusion constant $v_{0}=4 \pi e^{4}(\ln \Lambda) n_{\mathrm{H}} /\left(m^{2} v^{3}\right), x=$ $v^{2} / v_{\mathrm{te}}^{2}, \psi(x)=P(3 / 2, x), \psi^{\prime}(x)=2(x / \pi)^{1 / 2} \exp (-x)$, the electron thermal speed $v_{\mathrm{te}}=\left(2 k_{\mathrm{B}} T / \mathrm{m}\right)^{1 / 2}$, and $P$ is the incomplete gamma function (e.g., Press et al. 1986, p. 160). In the diffusion constant, the electron charge $e$ and the density $n_{\mathrm{H}}$ both have cgs units, and $\ln \Lambda$ is the Coulomb logarithm (taken to be 18 throughout the paper). An approximate diffusion coefficient is given by Spitzer (1962, p. 132). Using Huba's notation,

$$
D_{\mathrm{C}}(E)=4 v_{0} \frac{\psi(x)}{x} E^{2} .
$$

We show in Figure 1 the Coulomb convection and diffusion coefficients for electron-electron collisions in a $\mathrm{H}$ plasma of temperature $3 \times 10^{6} \mathrm{~K}$ and density of $10^{10} \mathrm{~cm}^{-3}$. The magnetic field is only used to normalize time. Below the thermal energy $E_{T}=k_{\mathrm{B}} T=5 \times 10^{-4} m c^{2}$, the convection coefficient is positive, and electrons systematically gain energy as a result of collisions. Above $E_{T}$, this coefficient is negative and electrons systematically lose energy.

The validity of these Coulomb convection and diffusion coefficients can be tested by examining the evolution of an initial electron distribution. Specifically, (1) an initial electron distribution with an average electron energy $\langle E\rangle_{0}$

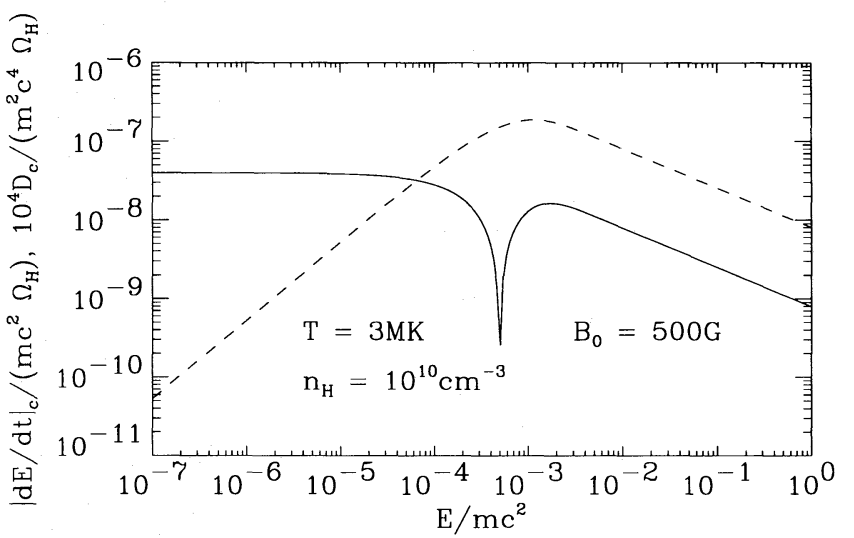

FIG. 1.-The Coulomb convection (solid line) and diffusion (dashed line) coefficients for an electron in the presence of a thermal electron background distribution of temperature $3 \times 10^{6} \mathrm{~K}$ and density $10^{10} \mathrm{~cm}^{-3}$. The magnetic field is specified to normalize time, and $\Omega_{\mathrm{H}}=4.79 \times 10^{6} \mathrm{~s}^{-1}$. Both coefficients are directly proportional to $n_{\mathrm{H}}$.

should relax to a Maxwellian distribution of the same average energy or one with $E_{\mathrm{T}}=2\langle E\rangle_{0} / 3$, and (2) an initially Maxwellian distribution should remain Maxwellian. As an example, in Figure 2 we place $10^{10}$ electrons $\mathrm{cm}^{-3}$ at an energy of $7.5 \times 10^{-4} \mathrm{mc}^{2}$. As such a distribution should evolve to a Maxwellian with $T=3 \times 10^{6} \mathrm{~K}$; we use the coefficients given in Figure 1 and numerically solve equation (2.3) (see $\S 2.3$ ). Panel $a$ shows the evolution from time $t=0$ to $10^{4} T_{\mathrm{H}}$, where $T_{\mathrm{H}}=\Omega_{\mathrm{H}}^{-1}$. The solid lines denote the distribution at 10 times during this interval. We see that the low-energy part of the distribution grows rapidly, whereas the high-energy tail suffers a much more gradual evolution. In panel $b$ we show the distribution from $t=10^{4}-10^{5} T_{\mathrm{H}}$. At these times both the low- and high-energy parts evolve slowly. In panel $c$ the simulation is continued and $t=$ $10^{5}-10^{6} T_{\mathrm{H}}$. Here the low-energy distribution has almost reached its equilibrium value, while the tail is still growing slightly. Beyond $t=10^{6} T_{\mathrm{H}}$, the spectrum suffers no further appreciable evolution. In panel $d$ we compare the distribution at $t=10^{6} T_{\mathrm{H}}$ with a Maxwellian distribution with $T=3 \times 10^{6} \mathrm{~K}$ or $E_{T}=5 \times 10^{-4} m c^{2}$. The agreement is excellent for all but the highest energies $\left(E \gtrsim 18 E_{\mathrm{T}}\right)$ in the tail, a result similar to that obtained by a more rigorous treatment using the Landau form of the Fokker-Planck equation (Macdonald, Rosenbluth, \& Chuck 1957). As a result of this and other similar tests, we conclude that this treatment of Coulomb collisions is very accurate.

\subsection{The Wave Diffusion Equation}

As a result of wave steepening, spectral energy will cascade to higher frequencies and wavenumbers, and we describe such a cascade by a diffusion equation in wavenumber space (Zhou \& Matthaeus 1990). Zhou \& Matthaeus sought to provide a simple framework for turbulence evolution in a variety of space physics problems, and this framework can be readily applied to our study of electron acceleration. The kinetic equation for a three-dimensional spectral density $\hat{W}_{T}(\boldsymbol{k})$ (wave energy density per unit volume of wavenumber space) is the usual conservation equation $\partial \hat{W}_{T}(\boldsymbol{k}) / \partial t=-\nabla_{\boldsymbol{k}} \cdot \boldsymbol{F}(\boldsymbol{k})$, where the flux $\boldsymbol{F}=-D \boldsymbol{\nabla}_{\boldsymbol{k}} \hat{W}_{T}(\boldsymbol{k})$, the diffusion coefficient in wavenumber space $D=k^{2} / \tau_{s}$, and the spectral energy transfer time is $\tau_{s}$. For isotropic turbulence, we can define a one-dimensional spectral density $W_{T}(k)=4 \pi k^{2} \hat{W}_{T}(\boldsymbol{k})$, and the diffusion equation simplifies to 

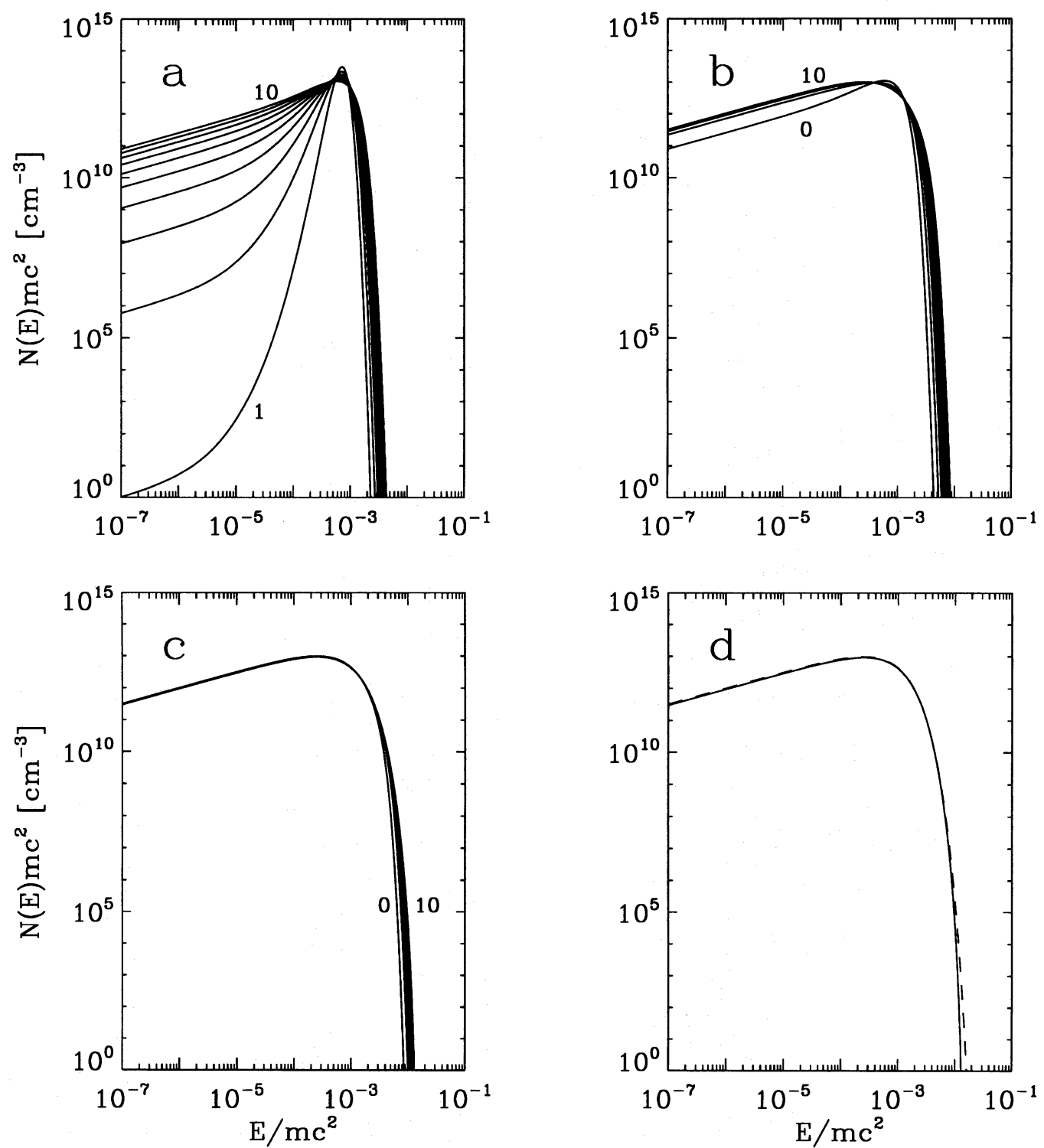

Fig. 2.-The evolution of a $\delta$-function electron distribution to a Maxwellian. (a) The electron distribution at times $t=10^{3} n T_{\mathrm{H}}$, where $n=1, \ldots, 10$. The value of $n$ is given by the numbers next to the curves. (b) The distribution at times $t=\left(10^{4}+9 \times 10^{3} n\right) T_{\mathrm{H}}$, where $n=0, \ldots, 10$. (c) The distribution at times $t=\left(10^{5}+9 \times 10^{4} n\right) T_{\mathrm{H}}$, where $n=0, \ldots, 10$. (d) Comparison of the distribution at $t=10^{6} T_{\mathrm{H}}$ (solid line) with a Maxwellian having the same average electron energy (dashed line). Time is normalized by taking $B_{0}=500 \mathrm{G}$, so that $T_{\mathrm{H}}=2.1 \times 10^{-7} \mathrm{~s}$.

$$
\frac{\partial W_{T}}{\partial t}=\frac{\partial}{\partial k}\left[k^{2} D \frac{\partial}{\partial k}\left(k^{-2} W_{T}\right)\right]-\gamma W_{T}+S,
$$

where we have included a term for the damping of the waves by the energetic electrons and a term $S$ for wave energy injection.

The diffusion coefficient depends upon the cascade phenomenology. In the Kolmogorov treatment, the spectral energy transfer time at a particular wavelength $\lambda$ is the eddy turnover time $\lambda / \delta v$, where $\delta v$ is the velocity fluctuation of the wave. In the Kraichnan treatment, the transfer time is longer by a factor of $v_{\mathrm{A}} / \delta v$. Both phenomenologies are further discussed in Zhou \& Matthaeus (1990) and yield

$$
D= \begin{cases}C^{2} v_{\mathrm{A}} k^{7 / 2}\left[\frac{W_{T}(k)}{2 U_{B}}\right]^{1 / 2}, & \text { (Kolmogorov), } \\ C^{2} v_{\mathrm{A}} k^{4}\left[\frac{W_{T}(k)}{2 U_{B}}\right], & \text { (Kraichnan), }\end{cases}
$$

where $C^{2}$ is the Kolmogorov constant that we take equal to unity. Upon substituting these diffusion coefficents into equation (2.6), and assuming a steady state with no damping, we obtain $W_{T}=W_{0} k^{-s}$, where $s=5 / 3$ for the Kolmogorov case and $s=3 / 2$ for the Kraichnan phenomenology. The diffusion equation in either case is nonlinear. While the Kolmogorov phenomenology should be appropriate for strong turbulence, it appears (as discussed in Miller \& Roberts 1995) that it is also better than the Kraichnan phenomenology for weak turbulence and thus more appropriate for this study. However, we consider both cases in this paper for completeness.

The damping rate $\gamma$ can be determined by conservation of energy. Employing the boundary conditions $N(\infty)=0$ and $D(E=0)=0$, we find from equation (2.3) that the volumetric energy gain rate of the particles $\dot{U}_{p}=$ $\int_{0}^{\infty} d E N(E) A(E)$, which is expected from the fact that $A(E)$ is the systematic acceleration rate. Requiring that $\dot{U}_{p}=-\dot{U}_{w}$, where the volumetric energy loss rate of the waves $\dot{U}_{w}=$ 
$-\int_{0}^{\infty} d k \gamma(k) W_{T}(k)$, and using equation (2.4a), we find that

$$
\gamma(k)=\Omega_{\mathrm{H}} \frac{\pi}{4}\left(\frac{v_{\mathrm{A}}}{c}\right)^{2} \frac{m c^{2}}{U_{B}}\left(\frac{c k}{\Omega_{\mathrm{H}}}\right) \int_{0}^{\infty} d E N(E)\left(\frac{p}{m c}\right) \mathrm{G}(v) .
$$

The damping rate is directly proportional to $k$, so that large-wavelength waves will suffer negligible damping even though the energetic electron spectrum may extend to very high energies. On the other hand, for sufficiently small wavelengths, the waves will be dissipated rapidly on the electrons. We thus expect a classical inertial and dissipation range to form in the spectral density.

We assume for simplicity that turbulence is deposited at a single wavelength $\lambda_{i}$. When turbulence is injected over time, we also assume that the injection rate is constant from time $t=0$ to $t_{f}$. Hence, $S=Q H\left(t_{f}-t\right) \delta\left(k-k_{i}\right)$, where $Q$ is the rate of wave energy density deposition, $H$ is the step function, and the injection wavenumber $k_{i}=2 \pi / \lambda_{i}$. If turbulence is present at $t=0$, then $W_{T}=U_{T i} \delta\left(k-k_{i}\right)$ initially, where $U_{T i}$ is the initial wave energy density.

\subsection{Method of Solution}

Electron acceleration and wave evolution are thus described by the two coupled partial differential equations (2.3) and (2.6), the latter of which is also nonlinear. To solve this system, we first transform to dimensionless variables, the use of which was already suggested by the form of the expressions for $D(p), A(E)$, and $\gamma$. Specifically, normalized momentum $\tilde{p}=p / m c$, kinetic energy $\widetilde{E}=E / m c^{2}$, speed $\tilde{v}=$ $v / c$, wavenumber $\tilde{k}=c k / \Omega_{\mathrm{H}}$, and time $z=t / T_{\mathrm{H}}$, where $T_{\mathrm{H}}=$ $\Omega_{\mathrm{H}}^{-1}$. The wave spectral density $W_{T}$ is normalized to $U_{B}$, so that $\tilde{W}_{T}=\Omega_{\mathrm{H}} W_{T} /\left(c U_{B}\right)$. That is, $\tilde{W}_{T} d \tilde{k}$ is the total wave energy density, in units of $U_{B}$, in the normalized wavenumber interval $d \tilde{k}$ about $\tilde{k}$. Last, the volumetric wave energy density injection rate $Q=\widetilde{Q} U_{B} \Omega_{\mathrm{H}}$. In the following sections, we use both normalized and unnormalized variables, depending upon convenience.

The technique used to solve this system is similar to that used in Miller \& Roberts (1995). Since $N(E)$ will span many decades in energy, we transform to logarithmic energy derivatives in the Fokker-Planck equation by $\partial / \partial E=[1 /$ $E \ln 10)] \partial / \partial y$, where $y=\log E$. The resulting equation is then finite differenced according to the Crank-Nicholson scheme (e.g., Press et al. 1986, p. 635). At the low-energy grid point we take the convection and diffusion coefficients equal to zero, while at the maximum-energy grid point we neglect diffusion but include convection. This technique takes into account the convection of particles to higher energies and thus prevents particle "pile up" at the last grid point.

We transform to logarithmic derivatives in the wave diffusion equation as well, which is then also differenced with the Crank-Nicholson method. If the maximum wavenumber grid point is well inside the dissipation range, then the waves will be damped completely before reaching it and $W_{T}$ can be set to zero there. At the minimum-wavenumber grid point, we take $W_{T}=0$, which is sufficient to prevent a flux of wave energy to low wavenumbers. Each differential equation yields a tridiagonal system of linear equations, which can be readily solved for $N$ and $W_{T}$ at each grid point. The integrations over $E$ in the expression for the wave damping rate, and over $k$ for the calculation of $U_{T}$ and $\langle k\rangle$ appearing in the expressions for the convection and diffusion coeffi- cients, are performed using the extended trapezoidal rule. We can also employ iteration in stepping forward from time $t_{n}$ to the next time $t_{n+1}$. In our scheme, coefficients at time $t_{n+1}$ are first assumed to be equal to those at time $t_{n}$. The quantities $N$ and $W_{T}$ are then stepped forward and used to find first-order corrections to the coefficients at $t_{n+1}$, which allows us to make the time step again with more accurate estimates of the coefficients at $t_{n+1}$. This process can be repeated an arbitrary number of times. We do not always need to employ iteration, but in those cases where we do, we find that one iteration is sufficient.

This overall scheme is very accurate and efficient. Adaptive time steps are not necessary, and we use a fixed time step in a given run. We incorporate many diagnostic features in the code as well, determining the extent of energy conservation and particle number, for example. The code also has the capability to restart from a previous run with a different time step, which is useful for examining the detailed behavior of the system in a small time interval of interest, such as when $\langle k\rangle$ becomes large enough to start accelerating electrons out of the tail. All simulations are performed with 30 grid points per decade in both $E$ and $k$ space and can be done on a workstation or fast personal computer.

\section{RESULTS}

We take the ambient $\mathrm{H}$ density and magnetic field to be $10^{10} \mathrm{~cm}^{-3}$ and $500 \mathrm{G}$, respectively. In an actual flare plasma, the initial temperature $T$ must be $\approx 3 \times 10^{6} \mathrm{~K}$ in order to account for the observed enhancements of heavy ions in the interplanetary particles (Reames et al. 1994), and so this is the temperature we assume for the initially Maxwellian electron distribution. These values imply that $T_{\mathrm{H}}=$ $2.09 \times 10^{-7} \mathrm{~s}, U_{B}=9.9 \times 10^{3} \mathrm{ergs} \mathrm{cm}^{-3}, v_{\mathrm{A}}=0.036 c$, and $v_{\mathrm{te}}=0.032 c$.

\subsection{Case 1}

In this trial, we consider a temporally extended injection of waves at $\lambda_{i}=1600 \mathrm{c} / \Omega_{\mathrm{H}}$, with $Q=4 \times 10^{-10} U_{B} \Omega_{\mathrm{H}}$ and $t_{f}=3 \times 10^{6} T_{\mathrm{H}}$. There is no turbulence present initially, and we employ the Kolmogorov phenomenology.

We show in Figure 3 the evolution of the turbulence from $t=0$ to $4 \times 10^{5} t_{\mathrm{H}}$. During this time the waves cascade to higher $k$, but the mean wavenumber $\langle k\rangle$ of the spectral density is not large enough for the waves to overcome Coulomb collisions and accelerate electrons out of the background. Consequently, the electron distribution remains a Maxwellian. In Figure $3 a$ we give the spectral density at 10 times during this interval, and the formation of a power-law spectrum with a $-5 / 3$ slope is evident at the later times. The spectral density at the injection wavenumber quickly reaches equilibrium, during which time the volumetric rate of wave energy injection $\widetilde{Q}$ must equal the volumetric rate at which this energy is transferred to smaller scales $-\tilde{k}^{2} \tilde{D} \partial\left(\tilde{k}^{-2} \tilde{W}_{T}\right) / \partial \tilde{k}$. For a Kolmogorov spectrum $\tilde{W}_{T}=\tilde{W}_{0} \tilde{k}^{-5 / 3}$, and we see from this condition and equation (2.7) that

$$
\tilde{W}_{0}=\left(\frac{3 \sqrt{2} \tilde{Q}}{11 \tilde{v}_{\mathrm{A}}}\right)^{2 / 3} .
$$

Note the important property that the normalization of the spectral density depends only upon the injection rate and not on the injection wavenumber. From equation (3.1), $\tilde{W}_{0}=2.6 \times 10^{-6}$, which is in excellent agreement with the 

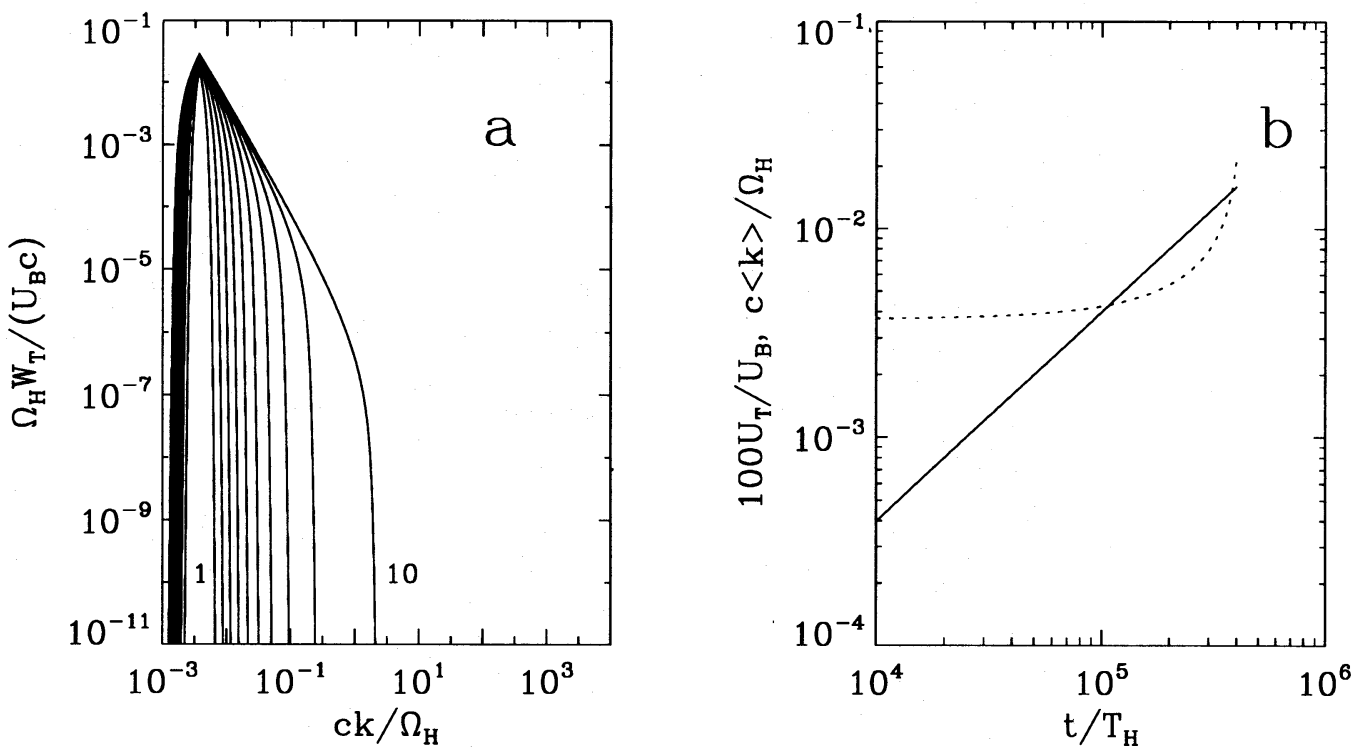

Fig. 3. - Case 1 evolution from $t=0$ to $4 \times 10^{5} T_{\mathrm{H}}$. (a) Wave spectral density at times $t_{n}=4 \times 10^{4} n T_{\mathrm{H}}$, where $n=1, \ldots, 10$. (b) Total energy density of the turbulence $U_{T}$ (solid line). Mean wavenumber $\langle k\rangle$ (dotted line).

simulation value near $t=4 \times 10^{5} T_{\mathrm{H}}$ and at subsequent times while the turbulence is still being injected. In Figure $3 b$, we show the total energy density of the turbulence $U_{T}$ and the mean wavenumber during this time. As there is no dissipation, $U_{T}=Q t$. The mean wavenumber does not have a simple analytical expression but increases by a factor of $\approx 5$ during this interval.

This time interval marks the end of pure cascading, and shortly afterwards $\langle k\rangle$ becomes large enough to accelerate electrons. This is illustrated in Figure 4. Figures $4 a$ and $4 b$ are the electron distribution and spectral density in the small time interval from $t=4 \times 10^{5}$ to $5 \times 10^{5} T_{\mathrm{H}}$, in which a power-law spectral density is established at nearly all $k$ and a nonthermal electron tail begins to form. Specifically, the electron distribution remains a Maxwellian until $t=$ $4.1 \times 10^{5} T_{\mathrm{H}}$. At $4.2 \times 10^{5} T_{\mathrm{H}}$, a power-law spectral density is established at all but the largest $k$. At this point the mean wavenumber rapidly increases and the acceleration rate becomes large enough to energize electrons out of the thermal background. The timescale $\tilde{\tau}_{k}$ for power-law formation at all $k$ will be approximately the turnover time of the largest scale fluctuation $\tilde{k}_{i}^{2} / \widetilde{D}\left(\tilde{k}_{i}\right)$ and is given by

$$
\tilde{\tau}_{k}=\sqrt{2} \tilde{v}_{\mathrm{A}}^{-1} \tilde{k}_{i}^{-2 / 3} \tilde{W}_{0}^{-1 / 2} .
$$

The cascade time of $9.7 \times 10^{5} T_{\mathrm{H}}$ from this equation overestimates that seen from the simulation by about a factor of 2 , indicating that an appreciable amount of wave energy can be transferred to smaller scales before a complete turnover of the largest scale.

Figures $4 c$ and $4 d$ show $N$ and $W_{T}$ from $t=5 \times 10^{5}$ to $10^{6} T_{\mathrm{H}}$. Here, the spectral density is in equilibrium (except for $k<k_{i}$ ) and shows essentially no deviation from its power-law form. The nonthermal tail of the electron distribution progressively hardens, being approximately proportional to $E^{-10}$ between 20 and $100 \mathrm{keV}$ at $5 \times 10^{5} T_{\mathrm{H}}$ and reaching $E^{-2.9}$ at $10^{6} T_{\mathrm{H}}$. During this time the total number density $N(>20 \mathrm{keV})$ of electrons above $20 \mathrm{keV}$ achieves a physically meaningful value of $\approx 10^{7} \mathrm{~cm}^{-3}$, and the specific acceleration rate of electrons $F(>20 \mathrm{keV})$ above $20 \mathrm{keV}$ reaches its maximum value of more than $10^{8} \mathrm{~cm}^{-3}$ $\mathrm{s}^{-1}$ (see Fig. 6a). The electron distribution and spectral density for the remainder of the time that waves are injected are shown in Figures $4 e$ and $4 f$. The main feature of the electron distribution is the pronounced flattening between $\approx 2 \times 10^{-3}$ and $\approx 2 \times 10^{-2} m c^{2}$, which is due to the rapid convective nature of the acceleration process and the relatively slow replenishment of electrons from lower energies. Hardening occurs at all energies, however, and between 20 and $100 \mathrm{keV}$ the distribution is proportional to $E^{-1.2}$ at $3 \times 10^{6} T_{\mathbf{H}}$. At this time there is also a significant number of electrons above $5 \mathrm{MeV}$. The spectral density remains a power law except at $c k / \Omega_{\mathrm{H}} \gtrsim 100$, where damping by the energetic electrons causes it to turn over slightly.

The distribution and spectral density after the injection of waves has stopped is shown in Figure 5. Near $E \approx 10^{-2} m c^{2}$, the electron distribution evolves to almost a constant form but does not change appreciably at higher energies. The quantity $N(>20 \mathrm{keV})$ remains approximately constant, and $F(>20 \mathrm{keV})$ drops very rapidly to levels uninteresting for hard X-ray production. The spectral density decreases about uniformly at all wavenumbers. Figure 6 is a summary of the most relevant electron and wave information for the entire simulation. The greater than $20 \mathrm{keV}$ specific acceleration rate is greater than $5 \times 10^{7} \mathrm{~cm}^{-3} \mathrm{~s}^{-1}$ for about $1.6 \times 10^{6} T_{\mathrm{H}}$, and $N(>20 \mathrm{keV})$ reaches a final value of $\approx 4 \times 10^{7} \mathrm{~cm}^{-3}$. Figure $6 b$ illustrates the rapid increase of $\langle k\rangle$ at $\approx 4 \times 10^{5} T_{\mathrm{H}}$, along with its subsequent decline due to progressively stronger damping at high wavenumbers by the electrons. During the time electrons are accelerated, $U_{T} \lesssim 2 \times 10^{-4} U_{B}$, which confirms that the turbulence is weak and the waves are low amplitude. This can also be seen from the relative wave magnetic field $\delta B / B_{0}=$ $\left(\tilde{W}_{T}(\tilde{k}) \tilde{k} / 2\right)^{1 / 2}$, where the number 2 takes into account the equipartition of energy between the magnetic field and the background ion motion. The largest relative amplitude is at the injection wavelength, where it is $\approx 7.2 \times 10^{-3}$.

The effect of Coulomb collisions upon the acceleration process can be readily determined by setting $D_{\mathrm{c}}=$ $(d E / d t)_{c}=0$ in the simulation and comparing the results with those obtained above. We show in Figure 7 the results for case 1 parameters but without collisions. Qualitatively, one expects that the absence of collisional drag above the 

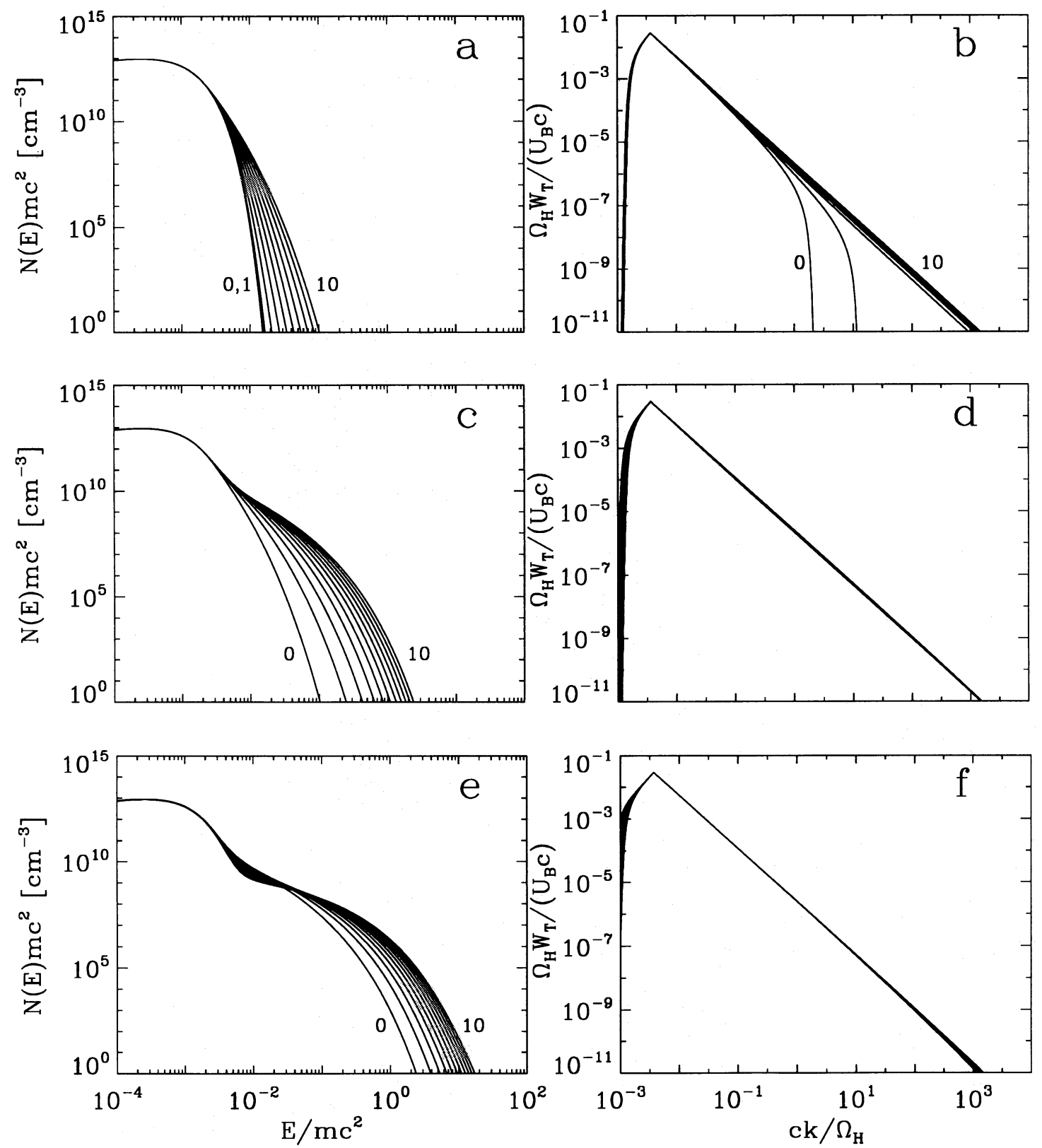

Fig. 4. - Case 1 evolution from $t=4 \times 10^{5}$ to $3 \times 10^{6} T_{\mathrm{H}}$. (a) and $(b)$ Evolution from $t=4 \times 10^{5}$ to $5 \times 10^{5} T_{\mathrm{H}}$. Electron distribution and wave spectral density are shown at times $t_{n}=\left(4 \times 10^{5}+10^{4} n\right) T_{\mathrm{H}}$ for $n=0, \ldots, 10 .(c)$ and $(d)$ Evolution from $t=5 \times 10^{5}$ to $10^{6} T_{\mathrm{H}}$. Electron distribution and wave spectral density are shown at times $t_{n}=\left(5 \times 10^{5} T_{\mathrm{H}}+5 \times 10^{4} n\right) T_{\mathrm{H}}$ for $n=0, \ldots, 10$. $(e)$ and $(f)$ Evolution from $t=10^{6}$ to $3 \times 10^{6} T_{\mathrm{H}}$. Electron distribution and wave spectral density are shown at times $t_{n}=\left(10^{6}+2 \times 10^{5} n\right) T_{\mathrm{H}}$ for $n=0, \ldots, 10$.
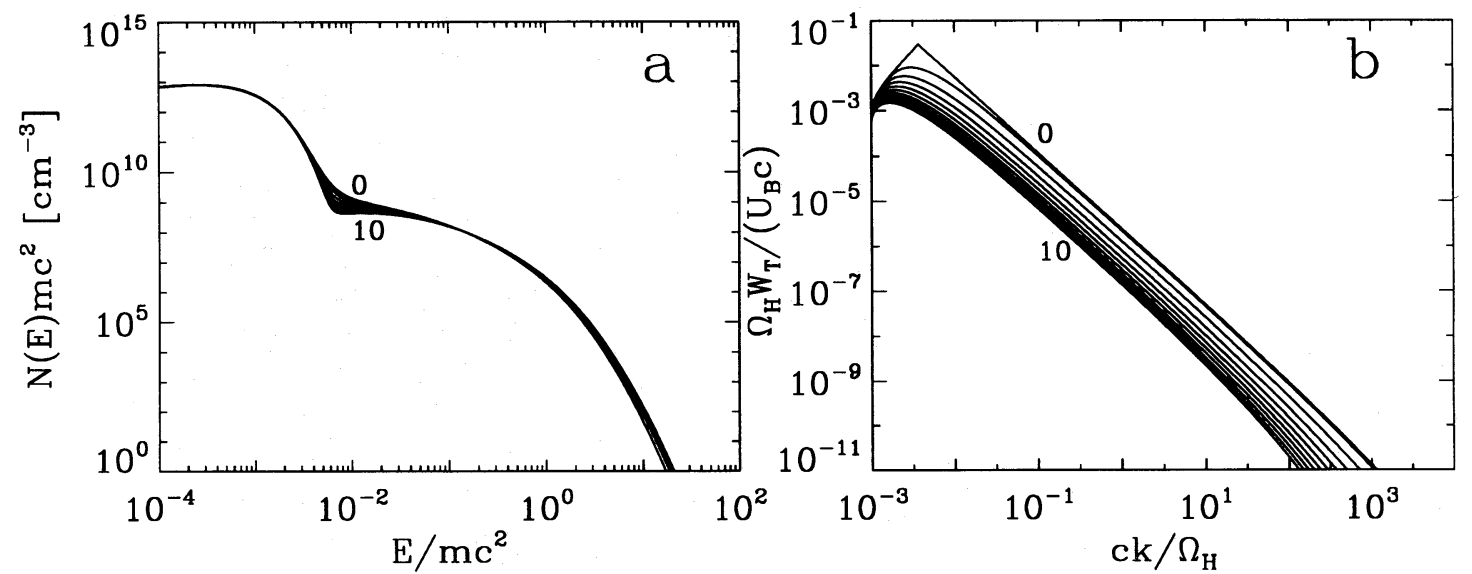

Fig. 5.-Case 1 evolution from $t=3 \times 10^{6}$ to $5 \times 10^{6} T_{\mathrm{H}}$. There is no further injection of turbulence. (a) Electron distribution at times $t_{n}=\left(3 \times 10^{6}+\right.$ $\left.2 \times 10^{5} n\right) T_{\mathrm{H}}$ for $n=0, \ldots, 10$. (b) Wave spectral density at the same 11 times. 

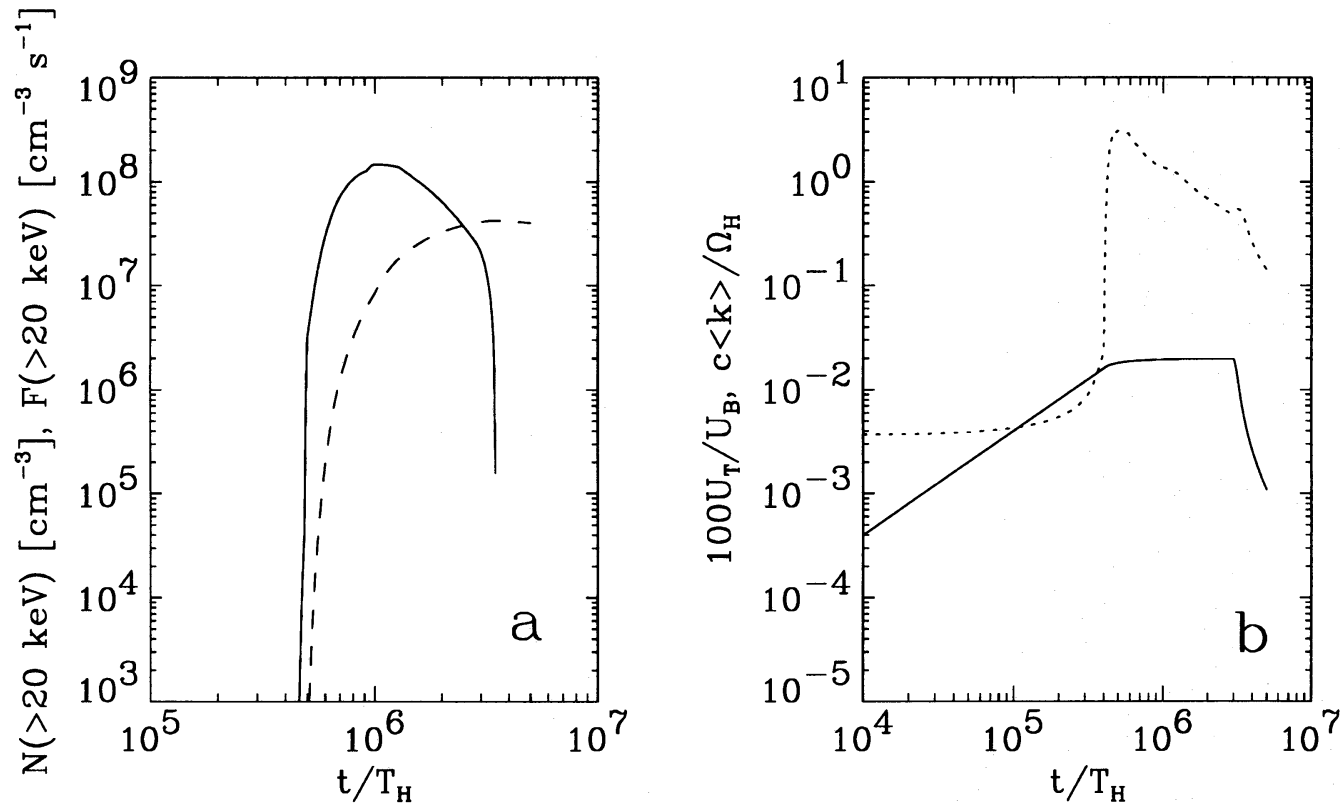

Fig. 6. - Case 1 summary. (a) The total number of electrons above $20 \mathrm{keV}$ (dashed line). The specific acceleration rate above $20 \mathrm{keV}$ (solid line). (b) Turbulence energy density $U_{T}$ (solid line). Turbulence mean wavenumber $\langle k\rangle$ (dotted line).

threshold energy would enable more electrons to be accelerated out of the Maxwellian and into high energies, and this is indeed the case. Panel $a$ shows that the total number density of electrons accelerated above $20 \mathrm{keV}$ is $\approx 10^{8}$ $\mathrm{cm}^{-3}$, which is about a factor of 2.5 times larger than the total number density with collisions. Similarly, the specific acceleration rate $F(>20 \mathrm{keV})$ is greater than $4 \times 10^{7} \mathrm{~cm}^{-3}$ $\mathrm{s}^{-1}$ for about $3 \times 10^{6} T_{\mathrm{H}}$, and greater than $10^{8} \mathrm{~cm}^{-3} \mathrm{~s}^{-1}$ for nearly $2.5 \times 10^{6} T_{\mathrm{H}}$. However, while the total number of accelerated electrons is greater without collisions, the average electron energy is smaller, since more electrons are absorbing energy from the same level of turbulence. In fact, the electron spectra is proportional to $E^{-2.2}$ between 20 and $100 \mathrm{keV}$ at $5 \times 10^{6} T_{\mathrm{H}}$, as opposed to nearly $E^{-1.2}$ from Figure $5 a$. The evolution of $U_{T}$ is essentially the same as before, but $\langle k\rangle$ without collisions decreases more rapidly after the injection of turbulence has stopped. Evidently, the larger number of electrons more than offsets the lower energies that they are accelerated to, and the damping rate is larger in this case than before.

\subsection{Case 2}

In the previous case, we assumed that the waves were injected at $\lambda_{i}=1600 \mathrm{c} / \Omega_{\mathrm{H}}$. Since the scale of turbulence generation is unknown at present for flares, we need to determine how this scale influences the results. The key for this is to notice that the acceleration rate is proportional to $U_{T}\langle k\rangle=\int_{0}^{\infty} d k k W_{T}(k)$. For a Kolmogorov phenomenology, this quantity is approximately $3 W_{0} k_{h}^{1 / 3}$, where $k_{h}$ is the wavenumber at which the power law turns over due to
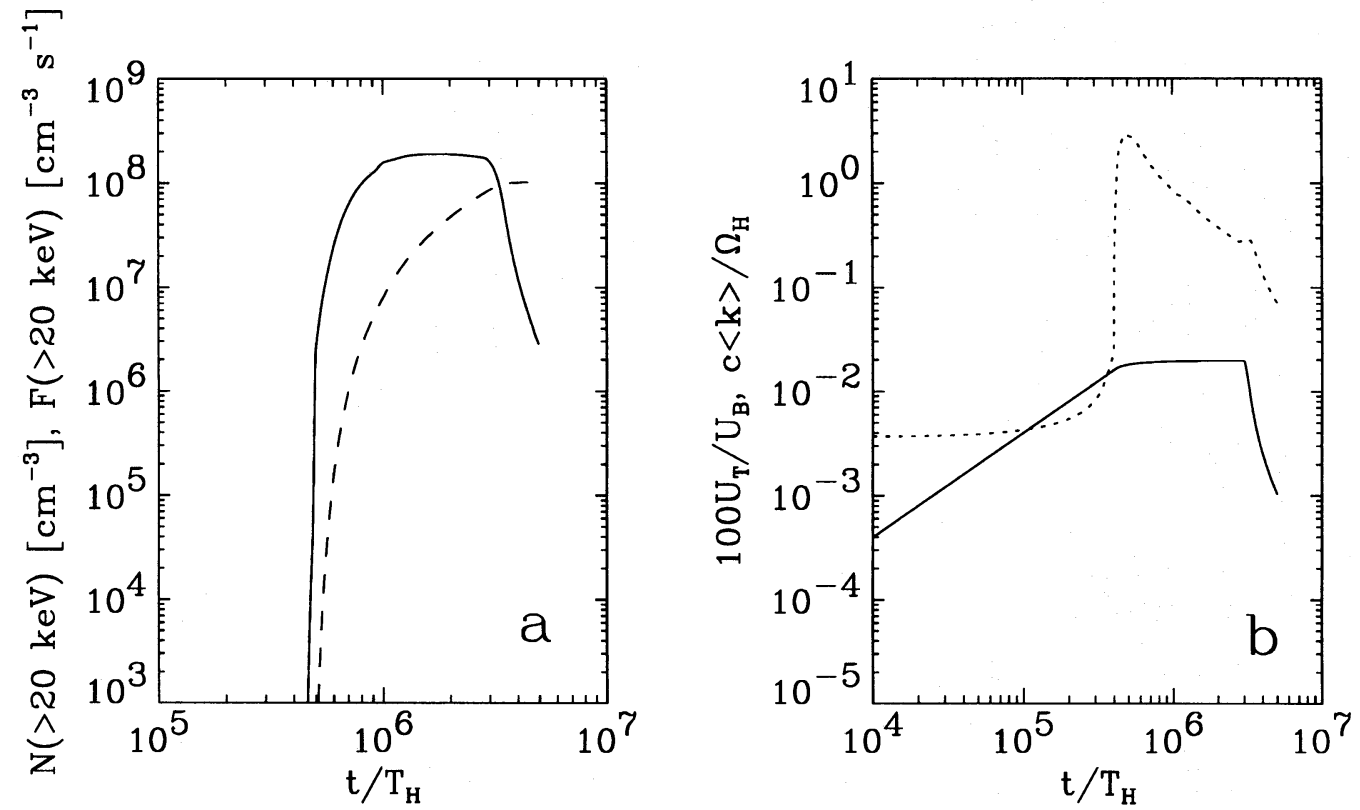

Fig. 7.-Same as Fig. 6 (case 1), except that Coulomb collisions have been neglected 
heavy damping by the accelerated electrons. Since $k_{h}$ should not depend upon the injection wavenumber $k_{i}$, as is the case with $W_{0}$, the acceleration rate when a steady state powerlaw spectral density is achieved is also independent of $k_{i}$. Therefore, we expect that the magnitude of electron quantities such as $N(>20 \mathrm{keV})$ and $F(>20 \mathrm{keV})$ should not vary appreciably with $k_{i}$, although their precise time dependence will (due to the change in the cascade time). This is an important result, since it means that a major unknown parameter is actually irrelevant (as long as $k_{i}<k_{h}$ ).

To quantitatively establish this conclusion, we consider another trial. In this trial all parameters are the same as those in case 1 , except that $\lambda_{i}=16000 \mathrm{c} / \Omega_{\mathrm{H}}$ (that is, $Q=$ $4 \times 10^{-10} U_{B} \Omega_{\mathrm{H}}, t_{f}=3 \times 10^{6} T_{H}$, there is no turbulence present initially, and we employ the Kolmogorov phenomenology). From equations (3.1) and (3.2), the cascade time is about $4 \times 10^{6} T_{\mathrm{H}}$, which is nearly 4 times longer than that in case 1 . We do not show results in the initial time interval when the waves are cascading to higher $k$ and the electron distribution is still a Maxwellian (they are similar to those in Fig. 3) but rather begin when the electrons are forming a nonthermal tail. We show in Figures $8 a$ and $8 b$ the electron distribution and wave spectral density at 11 times between $1.5 \times 10^{6}$ and $2 \times 10^{6} T_{\mathrm{H}}$. The electron distribution remains a Maxwellian until a power-law spectral density is formed out to large wavenumbers at about $1.95 \times 10^{6} T_{\mathrm{H}}$ and the mean wavenumber rapidly increases to $\approx 0.5 \Omega_{\mathrm{H}} / c$ (see Fig. $9 b$ ). At this point, a significant
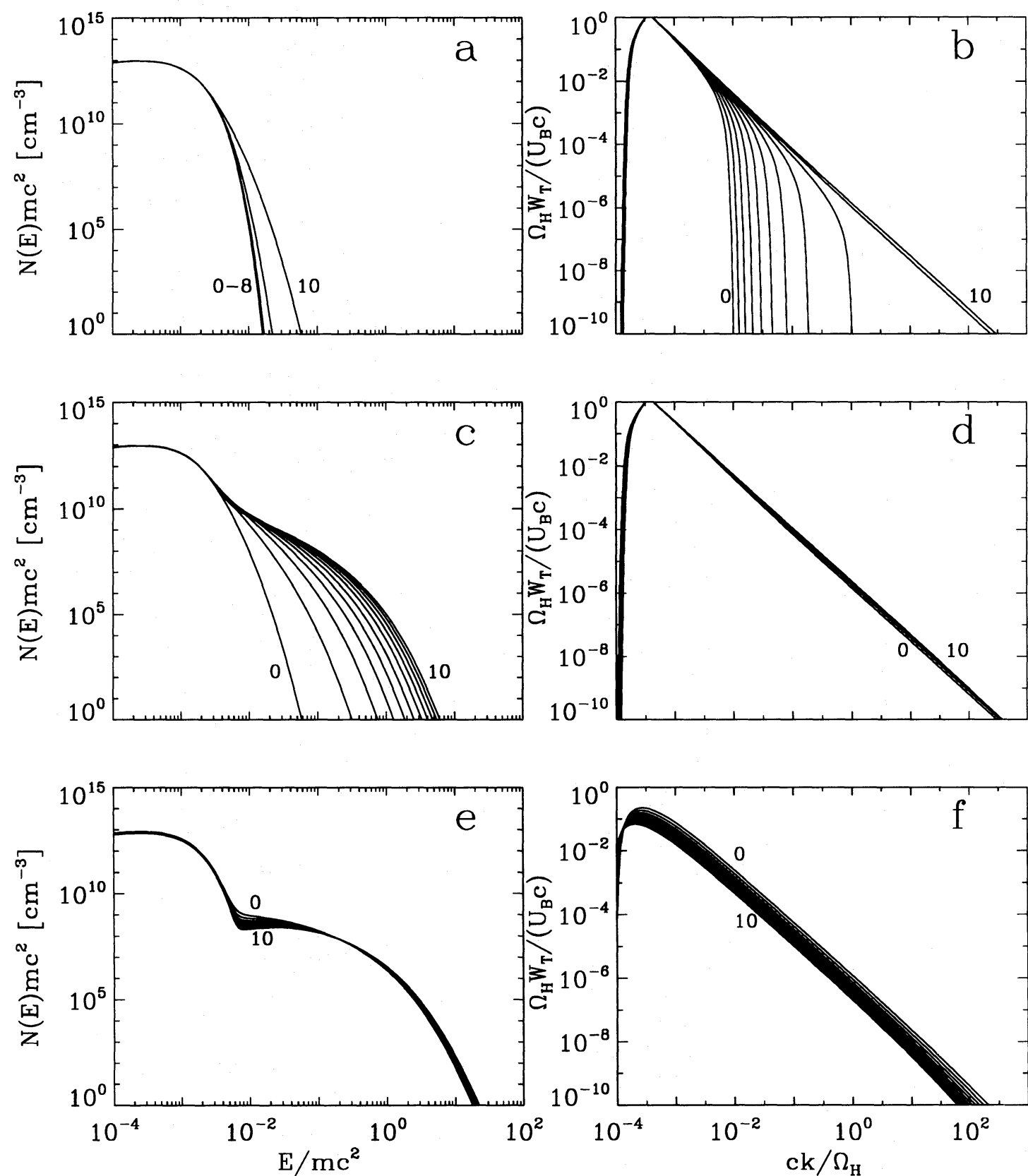

Fig. 8.-Case 2 evolution from $t=1.5 \times 10^{6}$ to $10^{7} T_{\mathrm{H}} \cdot(a)$ and $(b)$ Evolution from $t=1.5 \times 10^{6}$ to $2 \times 10^{6} T_{\mathrm{H}}$. Electron distribution and spectral density are shown at times $t_{n}=\left(1.5 \times 10^{6}+5 \times 10^{4} n\right) T_{\mathrm{H}}$ for $n=0, \ldots, 10 .(c)$ and $(d)$ Evolution from $t=2 \times 10^{6} T_{\mathrm{H}}$ to $3 \times 10^{6} T_{\mathrm{H}}$. Electron distribution and spectral density are shown at times $t_{n}=\left(2 \times 10^{6}+10^{5} n\right) T_{\mathrm{H}}$ for $n=0, \ldots, 10 .(e)$ and $(f)$ Evolution from $t=5 \times 10^{6}$ to $10^{7} T_{\mathrm{H}}$. Electron distribution and spectral density are shown at times $t_{n}=\left(5 \times 10^{6}+5 \times 10^{5} n\right) T_{\mathrm{H}}$ for $n=0, \ldots, 10$. 

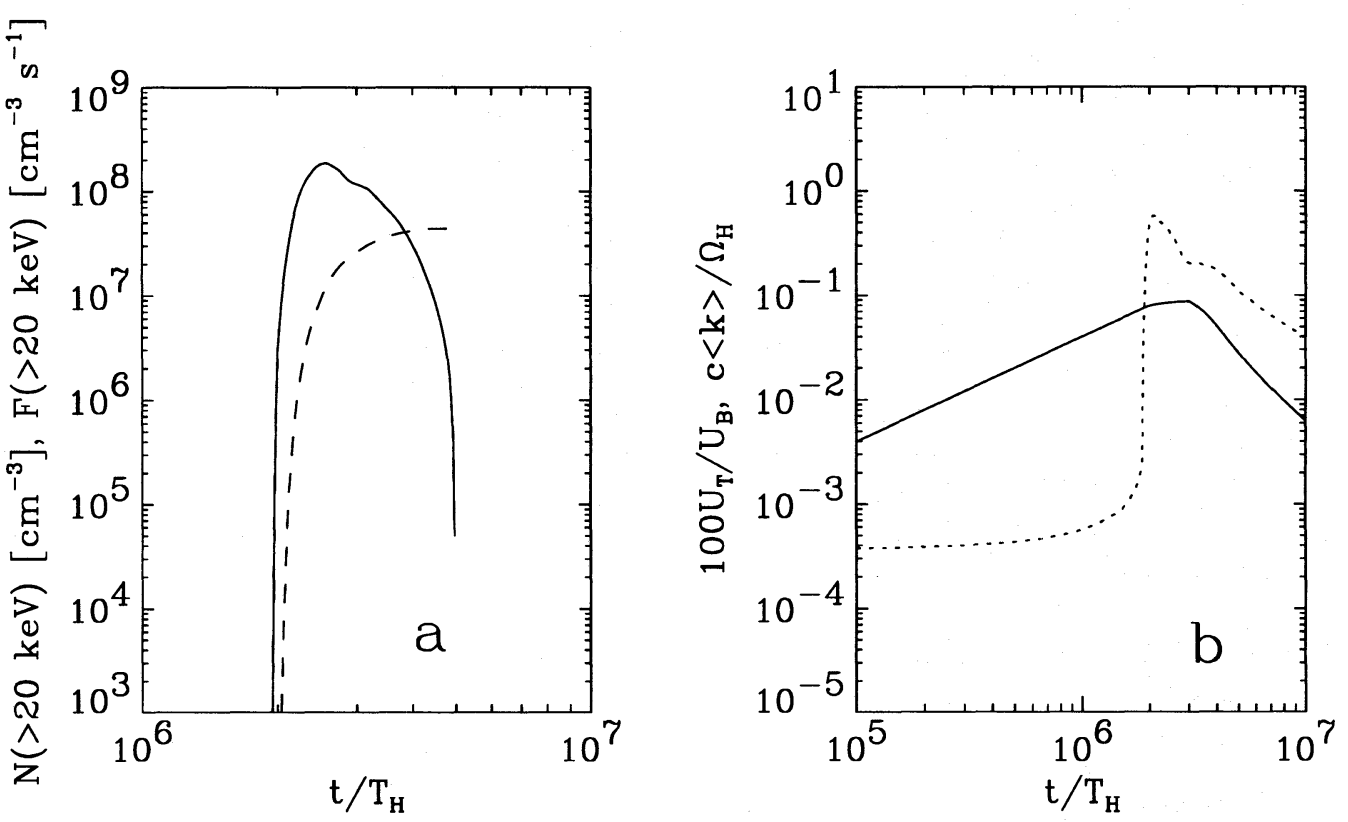

Fig. 9.-Case 2 summary. Notation is the same as in Fig. 6.

number of electrons above the threshold energy can overcome collisional drag and be energized out of the thermal distribution.

Figures $8 c$ and $8 d$ are the distribution and spectral density at 11 times from $2 \times 10^{6}$ to $3 \times 10^{6} T_{\mathrm{H}}$, at which time the injection of the turbulence stops. During this interval, the spectral density is in a quasi steady state, with $U_{T} \approx$ $8 \times 10^{-4} U_{B}$. Since the turbulence energy density $W_{T}\left(k_{i}\right) k_{i}$ in a wavenumber interval $k_{i}$ about $k_{i}$ is $\approx 4.7 \times 10^{-4} U_{B}$, $\delta B / B_{0}=1.5 \times 10^{-2}$ and all of the waves in the spectrum are small amplitude. The only significant evolution of $W_{T}$ is the decrease of $\langle k\rangle$ due to the accelerated electrons damping waves at high wavenumbers. The electron distribution steadily hardens, reaching $E^{-2}$ between 20 and $100 \mathrm{keV}$ at $3 \times 10^{6} T_{\mathrm{H}}$. The number density of electrons above $20 \mathrm{keV}$ also steadily increases, but the specific acceleration rate above $20 \mathrm{keV}$ reaches a maximum of more than $2 \times 10^{8} \mathrm{~cm}^{-3} \mathrm{~s}^{-1}$ at about $2.4 \times 10^{6} T_{\mathrm{H}}$. After the injection of the turbulence ceases, the spectral density uniformly decreases in a manner similar to that in Figure 5, while the electron distribution continues to harden at suprathermal energies. However, since the cascade time is relatively large, the turbulence stays at a relatively high level for a longer time, allowing the electron distribution to further evolve over a longer time as well. In fact, at $5 \times 10^{6} T_{\mathrm{H}}$, the electron distribution still shows appreciable evolution, as opposed to case 1 .

We do not show $N$ and $W_{T}$ from $3 \times 10^{6}$ to $5 \times 10^{6} T_{\mathrm{H}}$, since their behavior is similar to that in Figure 5. However, in Figures $8 e$ and $8 f$ we show these quantities from $5 \times 10^{6}$ to $10^{7} T_{\mathrm{H}}$. Aside from the very hard spectrum $\left(E^{-0.6}\right)$ between 20 and $100 \mathrm{keV}$, note a slight bump-on-tail feature near $\approx 5 \mathrm{keV}$. This is due to the systematic convection of electrons to higher energies (e.g., see also Fig. 3 or Ramaty 1979) in the absence of a correspondingly rapid replenishment from the thermal distribution. Beyond $10^{7} T_{\mathrm{H}}$, further evolution is small. This bump-on-tail feature is interesting and may be of importance in ${ }^{3} \mathrm{He}$ acceleration models (see $\S 4)$.

A summary of this simulation is given in Figure 9. The total number density of electrons above $20 \mathrm{keV}$ is the same as that in case 1 , and $F(>20 \mathrm{keV})$ is greater than $5 \times 10^{7}$ $\mathrm{cm}^{-3} \mathrm{~s}^{-1}$ for about $1.6 \times 10^{6} T_{\mathrm{H}}$, also the same as in case 1 . This supports our expectation that the essential electron results are independent of the scale at which waves are injected. This is not to say, however, that all results are identical. Specifically, the wave characteristics are quite different. The peak value of $c\langle k\rangle / \Omega_{\mathrm{H}}$ is about 0.6 in case 2 , as opposed to about 4 in case 1 , although this quantity has the same $e$-folding decay time after turbulence injection stops. The decrease of $\langle k\rangle$ in this case is offset by an increase of $U_{T}$, so that the product remains about the same.

\subsection{Case 3}

The last simulation established the fact that the scale at which the turbulence is generated does not appreciably affect the essential properties of electron acceleration. The next question is whether the timescale of the turbulence generation does. In the above cases, we have taken the injection to be over $3 \times 10^{6} T_{\mathrm{H}}$. Injection over longer times with the same injection rate $Q$ can yield sufficiently high electron fluxes, but the total amount of turbulence will be larger. Since we seek to establish the sufficient conditions under which the observed electron acceleration can occur, we want to keep the total amount of turbulence to a minimum. Hence, we consider injection over shorter times, and we thus take the limit of an impulsive deposition at $t=0$.

In this trial all parameters are the same as those in case 2 $\left(\lambda_{i}=16000 c / \Omega_{H} ;\right.$ the Kolmogorov phenomenology), except that the initial level of turbulence $U_{T i}$ at $\lambda_{i}$ is $1.2 \times 10^{-3} U_{B}$ and there is no subsequent injection of more wave energy. Hence, in the code at $t=0, \sum_{\mathrm{j}} \tilde{W}_{T}\left(k_{j}\right) \Delta \tilde{k}_{j}=\tilde{U}_{T i}$, where $k_{j}$ is a wavenumber grid point and $\Delta k_{j}=2.3 k_{j} \Delta \log k_{j}$ is the wavenumber interval about $k_{j}$. With 30 points per decade in $\log \tilde{k}$ space, we have that $\tilde{W}_{T}\left(k_{i}\right)=40$. This is larger than any value of $\tilde{W}_{T}$ encountered in the continuous injection cases, but $\delta B / B_{0}$ at the injection wavelength is about $9 \times 10^{-2}$, so that the waves are still small amplitude.

The wave energy is transferred to higher $k$ more quickly than in case 2 since its initial level is larger. Specifically, we 
approximate the cascade timescale $\tilde{\tau}_{k}$ by the eddy turnover time at the injection wavelength $k_{i}^{-1} / \tilde{\delta} v_{i}$, where $\tilde{\delta} v_{i}$ is the velocity fluctuation at $k_{i}$. Since $\delta v_{i} / v_{\mathrm{A}}=\delta B / B_{0}=\left(\widetilde{U}_{T i} / 2\right)^{1 / 2}$, we have that $\tilde{\tau}_{k} \approx 2.9 \times 10^{6}$. The time for a power-law spectral density to form in the simulation is about $8 \times 10^{5} T_{H}$. This is shorter than the estimate and is due to the fact that a power law can form before a complete turnover of the largest fluctuation. At the moment a power law is established, the normalization constant $\tilde{W}_{0}$ is given by $\tilde{U}_{T i} \approx$ $3 \tilde{W}_{0} \tilde{k}_{i}^{-2 / 3} / 2$. This is also an approximate expression, since by the time that the power law is formed, some wave energy has already flowed into the electrons. This condition predicts that $\widetilde{W}_{T}\left(k_{i}\right)=2$, in fair agreement with the simulations which yield a value of 1 .

We do not show electron distributions and spectral densities as in Figures 3-5 and 8 since their behavior is not significantly different. The main difference in the spectral density is that $W_{T}\left(k_{i}\right)$ is continually decreasing, as opposed to the earlier cases where it remained constant throughout most of the turbulence injection. The electron distribution at $3 \times 10^{6} T_{\mathrm{H}}$, which is when the bulk of the evolution is over, is similar to the electron distribution at the same time in Figure $4 e$. We give in Figure 10 a summary of this simulation, showing the pertinent electron and wave data as before. The total number of electrons above $20 \mathrm{keV}$ reaches about $6 \times 10^{7} \mathrm{~cm}^{-3}$ after $3 \times 10^{6} T_{\mathrm{H}}$, and the specific acceleration rate above $20 \mathrm{keV}$ remains above $5 \times 10^{7} \mathrm{~cm}^{-3} \mathrm{~s}^{-1}$ for about $1.2 \times 10^{6} T_{\mathrm{H}}$. The wave energy density remains approximately constant at its initial level until the mean wavenumber suffers a large increase to $\simeq 1.3 \Omega_{\mathrm{H}} / c$, at which point electrons are appreciably accelerated and both $U_{T}$ and $\langle k\rangle$ decrease in response.

\subsection{Case 4}

In this trial we consider cascading according to the Kraichnan phenomenology. The initial behavior of the system is similar to that obtained using a Kolmogorov phenomenology, but the longer cascade time results in qualitatively different spectral densities. Specifically, we consider a temporally extended injection of waves at $\lambda_{i}=160 \mathrm{c} / \Omega_{\mathrm{H}}$, with $Q=4 \times 10^{-10} U_{B} \Omega_{\mathrm{H}}$ and $t_{f}=3 \times 10^{6} T_{\mathrm{H}}$. There is no turbulence present initially. Note the smaller scale at which the waves are generated (see $\S 4)$.

We show in Figures $11 a$ and $11 b$ the electron distribution and spectral density at 11 times from $t=0$ to $3 \times 10^{6} T_{\mathrm{H}}$, when the wave injection ceases. At about $6 \times 10^{5} T_{H}$, a nonthermal tail begins to form, and it progressively hardens as wave energy flows to smaller scales and the acceleration rate increases. The spectral density forms a power law up to some wavenumber $k_{h}$, above which the spectral density rapidly turns over and approaches zero. This turnover wavenumber steadily increases up to $2.4 \times 10^{6} T_{H}$, when there are a sufficient number of electrons at high energies to cause very strong damping at high $k$, which in turn leads to the high- $k$ part of the spectral density "snapping back" to lower $k$. This phenomenon also occurs for Kolmogorov cascading but to a much less noticeable extent and at significantly higher $k$ (cf. Figs. $4 b$ and $4 d$ with Fig. $11 b$ ). The reason why the effect is so pronounced here is that the cascade timescale is much longer and turbulence at high $k$ cannot be replenished from lower $k$ as rapidly as it is dissipated by the energetic electrons. This relatively slow spectral energy cascade also leads to the waves being confined to much lower $k$ than in the Kolmogorov case.

The cascade timescale and spectral density normalization can be estimated as with the Kolmogorov cases. Equating $\widetilde{Q}$ with the volumetric energy cascade rate $-\tilde{k}^{2} \tilde{D}$ $\partial\left(\tilde{k}^{-2} \tilde{W}_{T}\right) / \partial k$, assuming a Kraichnan spectrum $\tilde{W}_{T}=$ $\widetilde{W}_{0} k^{-3 / 2}$, and using $D$ from equation (2.7), we find that

$$
\tilde{W}_{0}=\left(\frac{4 \tilde{Q}}{7 \tilde{v}_{\mathrm{A}}}\right)^{1 / 2}
$$

at equilibrium. From equations (3.1) and (3.3), the normalization of the Kraichnan spectrum is greater than that for the Kolmogorov spectrum when $\widetilde{Q} \lesssim 0.3$, which is expected since spectral energy transfer is inhibited by the magnetic field in the Kraichnan phenomenology and cascading into
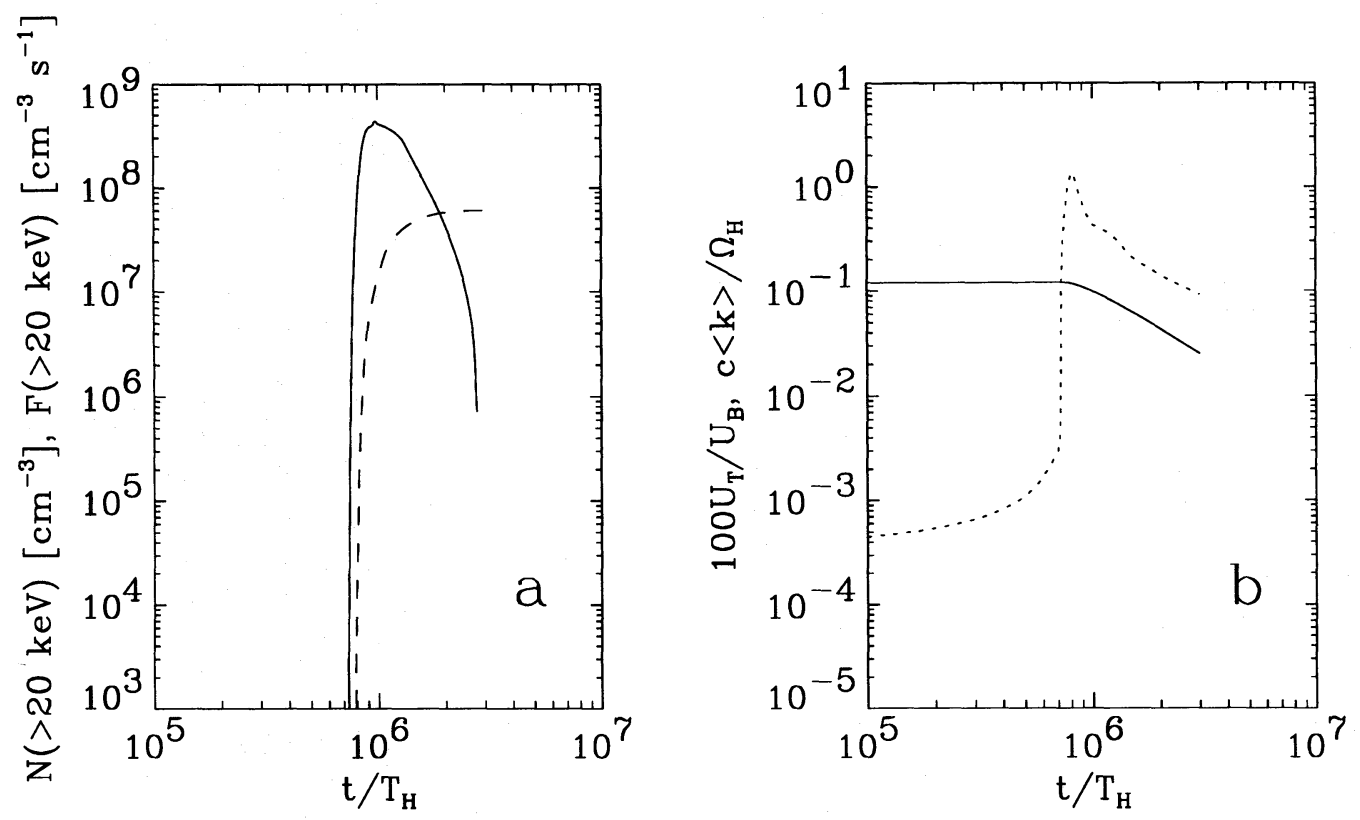

Fig. 10.-Case 3 summary. Notation is the same as in Fig. 6. 

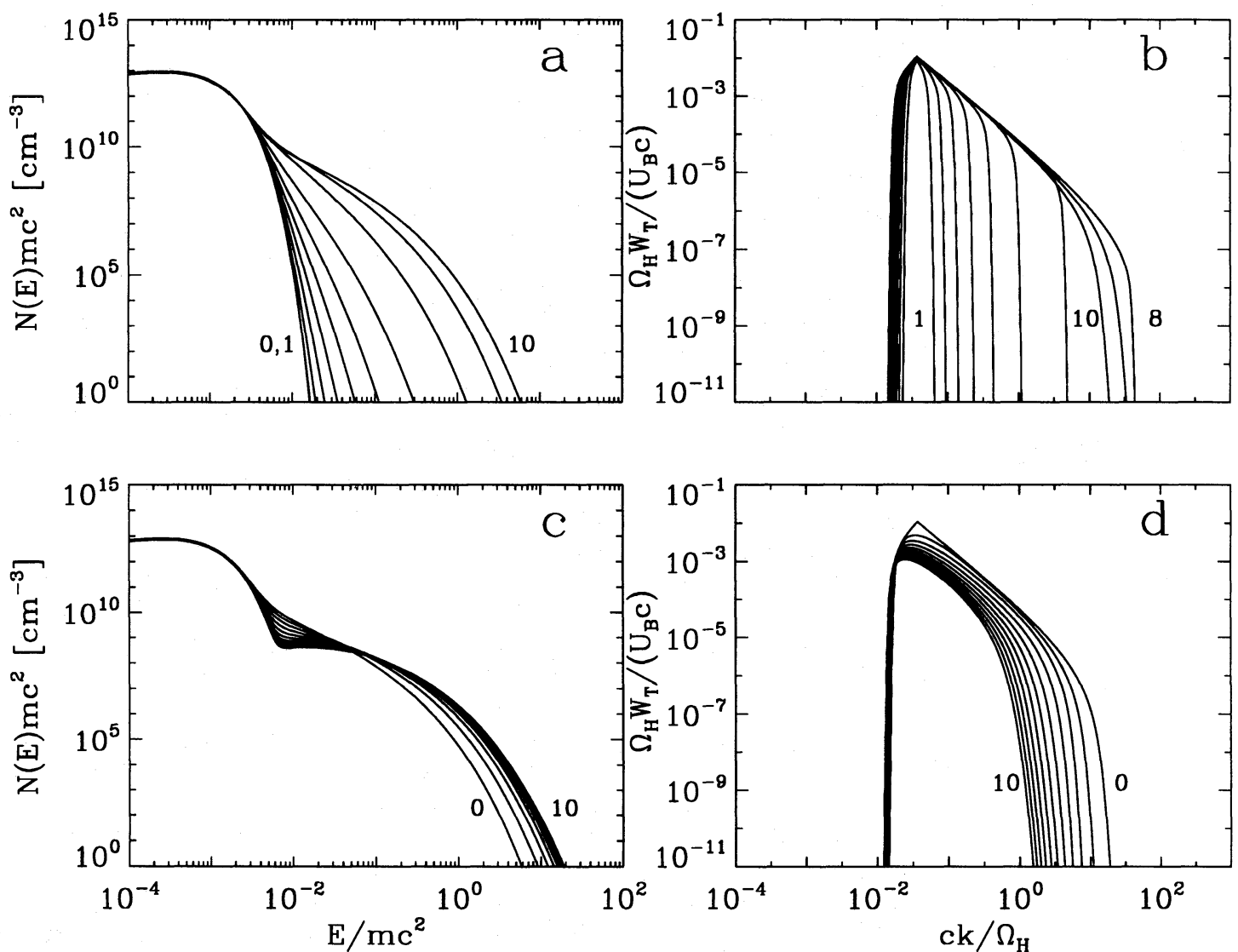

Fig. 11.-Case 4 evolution from $t=0$ to $7 \times 10^{6} T_{\mathrm{H}}$. (a) and $(b)$ Evolution from $t=0$ to $3 \times 10^{6} T_{\mathrm{H}}$. Electron distribution and wave spectral density are shown at times $t_{n}=3 \times 10^{5} n T_{\mathrm{H}}$ for $n=0, \ldots, 10$. (c) and $(d)$ Evolution from $t=3 \times 10^{6}$ to $7 \times 10^{6} T_{\mathrm{H}}$. Electron distribution and spectral density are shown at times $t_{n}=\left(3 \times 10^{6}+4 \times 10^{5} n\right) T_{\mathrm{H}}$ for $n=0, \ldots, 10$.

the dissipation range will not occur as quickly. The cascade timescale $\tilde{\tau}_{k r}$ is given by

$$
\tilde{\tau}_{k r}=2 \tilde{v}_{\mathrm{A}}^{-1} \tilde{k}_{i}^{-1 / 2} \tilde{W}_{0}^{-1},
$$

which is also typically greater than $\tilde{\tau}_{k}$ for the same reason. With $\widetilde{Q}=4 \times 10^{-10}$ and $\tilde{k}_{i}=3.93 \times 10^{-2}$, we have $\tilde{W}_{0} \approx$ $8 \times 10^{-5}$ and $\tau_{k r} \approx 3 \times 10^{6} T_{\mathrm{H}}$. From Figure $11 b$, these estimates are in very good agreement with those obtained from the simulation. At the injection wavelength, $\delta B / B_{0} \approx$ $1.4 \times 10^{-2}$, and the waves are low amplitude in this case as well.

By the time the wave energy injection stops, the electron distribution has an approximate $E^{-2}$ form between 20 and $100 \mathrm{keV}$. The evolution of the system from $t=3 \times 10^{6}$ to $7 \times 10^{6} T_{\mathrm{H}}$ is shown in panels $c$ and $d$. During this time, the electron distribution suffers the usual further development into a harder spectrum and the spectral density uniformly decreases due to damping. A summary of the relevant quantities is given in Figure 12. The total number of electrons accelerated to energies above $20 \mathrm{keV}$ is $\approx 4 \times 10^{7} \mathrm{~cm}^{-3}$, and the specific acceleration rate above this energy remains above $5 \times 10^{7} \mathrm{~cm}^{-3} \mathrm{~s}^{-1}$ for about $1.6 \times 10^{6} T_{\mathrm{H}}$. The mean wavenumber never exceeds $\sim 0.4 \Omega_{\mathrm{H}} / c$, due to the strong damping and the slow replenishment of wave energy.

\section{DISCUSSION}

Since the Fokker-Planck equation is in terms of the energy-differential electron density, while hard X-ray spectra give the rate of acceleration of energetic electrons, we need to assume a volume of the acceleration region in order to compare the two. We take this volume to be $10^{27}$ $\mathrm{cm}^{3}$, so that the basic observations we wish to account for are a specific acceleration rate above $20 \mathrm{keV}$ of $\approx 5 \times 10^{7}$ electrons $\mathrm{cm}^{-3} \mathrm{~s}^{-1}$ over $\approx 400 \mathrm{~ms}$ and thus a total number density of electrons above $20 \mathrm{keV}$ of $\approx 2 \times 10^{7} \mathrm{~cm}^{-3}$. Consider first the Kolmogorov cascading results. In cases 1-3, we have established that an injection of $12 \mathrm{ergs} \mathrm{cm}^{-3}$ on any scale less than $10^{8} \mathrm{~cm}$ and over any time interval less than $\approx 600 \mathrm{~ms}$ (which is at the upper limit of a burst duration) will yield an $F(>20 \mathrm{keV})$ of more than $5 \times 10^{7}$ $\mathrm{cm}^{-3} \mathrm{~s}^{-1}$ for $240-320 \mathrm{~ms}$ and an $N(>20 \mathrm{keV})$ of 4-6 $\times 10^{7}$ $\mathrm{cm}^{-3}$. Since the total number density of energetic electrons above $20 \mathrm{keV}$ is $2-3$ times that needed and the time over which $F(>20 \mathrm{keV})$ exceeds $5 \times 10^{7} \mathrm{~cm}^{-3} \mathrm{~s}^{-1}$ is consistent with our approximate fiducial value of $400 \mathrm{~ms}$, we conclude that this scenario can account for the hard X-ray burst observations. Of course, an injection of a larger amount of turbulence would also be permissible, but $12 \mathrm{ergs} \mathrm{cm}^{-3}$ is a sufficient quantity.

Generating the turbulence at lower wavenumbers will lead to a delay of the electron acceleration relative to the start of the injection but will not, as we have argued, appreciably affect the overall characteristics of the acceleration. While this is always true in our model, it is true only up to a point in an actual flare environment. The reason is that longer cascade times increase the importance of convective losses for the waves, which will eventually affect electron acceleration by decreasing the level of turbulence remaining in the region. In order for convective losses to have a small effect, (1) the cascade time must be smaller than the wave 

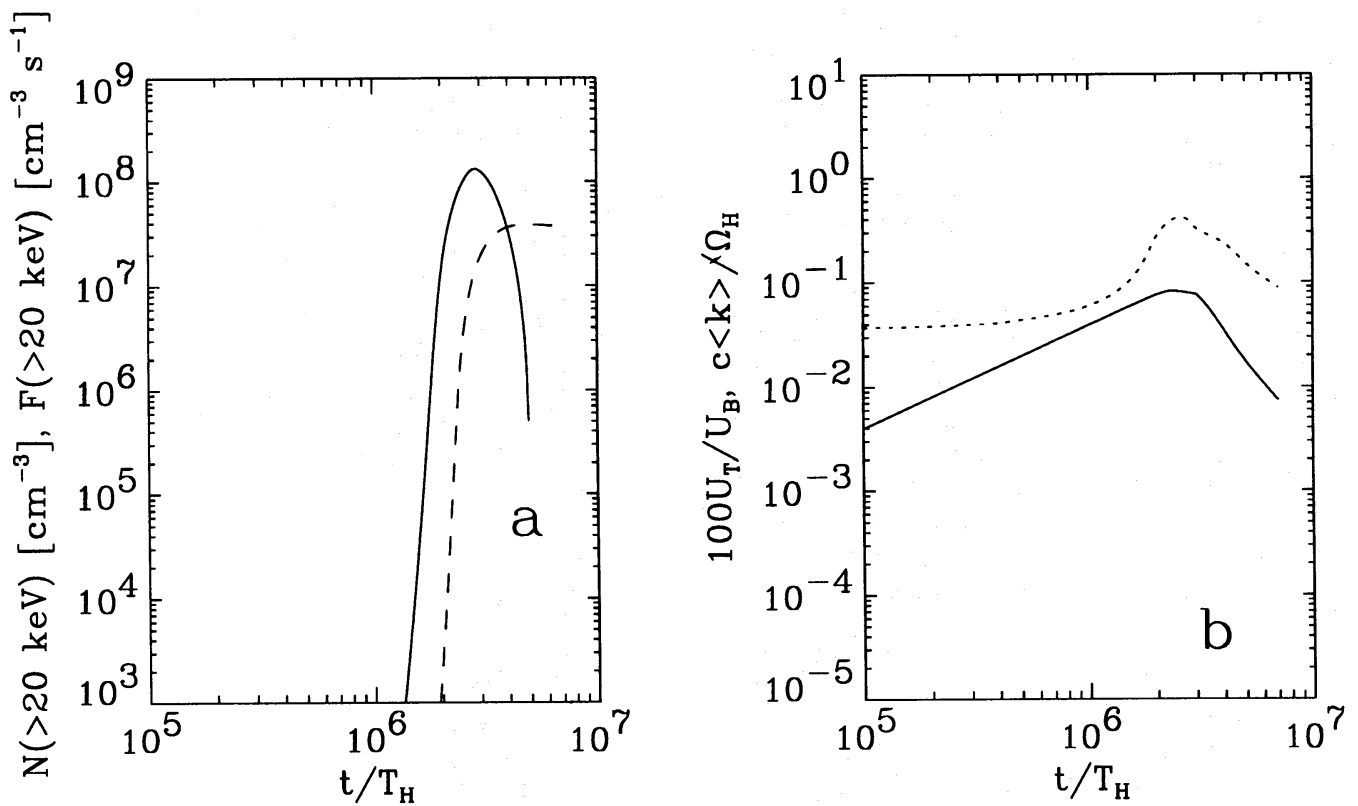

Fig. 12.-Case 4 summary. Notation is the same as in Fig. 6.

transit time $L / v_{\mathrm{A}}$, where $L$ is the scale size of the region that we take to be $\left(10^{27} \mathrm{~cm}^{3}\right)^{1 / 3}$ and (2) the time over which electron acceleration occurs must be less than $L / v_{\mathrm{A}}$. The second condition is automatically satisfied since we are already requiring acceleration over about $400 \mathrm{~ms}$, but the first is not. From the previous section, we see that condition (1) requires that $\tilde{k}_{i}^{2 / 3} \tilde{W}_{0}^{1 / 2}$ be greater than $8.9 \times 10^{-6}$ for extended injection or $\tilde{k}_{i}\left(\tilde{U}_{T i} / 2\right)^{1 / 2}$ be greater than $6.3 \times 10^{-6}$ for impulsive injection. Using $\widetilde{Q}=4 \times 10^{-10}$ and $\tilde{U}_{T i}=1.2 \times 10^{-3}$ as before, either condition implies that $\lambda_{i}$ needs to be smaller than $\approx 10^{8} \mathrm{~cm}$. However, larger quantities of turbulence would increase this upper limit accordingly.

It is of interest to consider acceleration timescales in addition to total numbers and specific acceleration rates above $20 \mathrm{keV}$. In the absence of the factor $\mathrm{G}(v)$ in the expression for the systematic acceleration rate $A(E)$ (as is the case in Fermi acceleration), this rate would be proportional to $E^{1 / 2}$ in the nonrelativistic regime and proportional to $E$ in the ultrarelativistic limit. However, as $\mathrm{G}(v)$ is an increasing function of $E$, we have instead that $A(E)$ is nearly proportional to $E$ from nonrelativistic to fully relativistic energies. Specifically, $A(E) \approx 4.5$ $\times 10^{-3} m c^{2} \Omega_{\mathrm{H}} \widetilde{U}_{T}\langle\tilde{k}\rangle \tilde{E}^{5 / 6}$ for $10^{-2} \leq E \leq 10^{2}$. Therefore, the acceleration time $\tau_{E}$ from $10^{-2} m c^{2}$ to $E$ is given by $1390\left(\tilde{E}^{1 / 6}-0.48\right)\left(\tilde{U}_{T}\langle\tilde{k}\rangle\right)^{-1} T_{\mathrm{H}}$. This expression is valid regardless of the cascading phenomenology or the precise shape of the spectral density and can be used with Figures 6 , 7,10 , or 12 to find acceleration timescales for a given wave energy density $U_{T}$ and mean wavenumber $\langle k\rangle$. To extend this result to lower initial energies, we have that $\tau_{E}$ from $E=7.8 \times 10^{-4} \mathrm{mc}^{2} \quad$ (corresponding to $v=1.1 v_{\mathrm{A}}$ ) to $E=10^{-2} m c^{2}$ is $4380\left(\tilde{U}_{T}\langle\tilde{k}\rangle\right)^{-1} T_{\mathrm{H}}$. This timescale is relatively large compared with those going from $10^{-2} m c^{2}$ to higher energies and is due to the steep decline of $\mathrm{G}(v)$ near the threshold for acceleration.

For example, when the power-law spectral density is first formed at $t \approx 5 \times 10^{5} T_{\mathrm{H}}$ in case 1 , we have from Figure 6 that $\widetilde{U}_{T}=2 \times 10^{-4}$ and $\langle\tilde{k}\rangle=4$, so that the timescale for acceleration up to $10^{-2} m c^{2}$ is nearly $1 \mathrm{~s}$. Since both $U_{T}$ and $\langle k\rangle$ subsequently decrease, the actual time is longer. As the product of $U_{T}$ and $\langle k\rangle$ for the other simulations is comparable to when a nonthermal tail begins to form, we see that transit-time damping does not typically yield extremely short acceleration times at low energies. Cast in this light, the process is not that efficient. However, the point is that enough ambient electrons are accelerated to $\approx 10^{-2} m c^{2}$ over a subsecond time interval to produce a sufficient flux to higher energies. Above $10^{-2} m c^{2}$, the acceleration times decrease greatly, and $\tau_{E}$ from $10^{-2}$ to $10^{-1} m c^{2}$ or $m c^{2}$ is $290\left(\tilde{U}_{T}\langle k\rangle\right)^{-1} T_{\mathrm{H}}$ or $720\left(\tilde{U}_{T}\langle k\rangle\right)^{-1} T_{\mathrm{H}}$, respectively. At the instant when the power law is formed, it takes only about 75 ms to go from $\approx 5$ to $50 \mathrm{keV}$ or about $180 \mathrm{~ms}$ to go from $\approx 5$ to $511 \mathrm{keV}$. Again, the actual times will be longer since the turbulence is being dissipated over the duration of the acceleration. We note that since this is a diffusive process, these are average times and some electrons are accelerated more rapidly, just as some are accelerated more slowly.

We thus expect the acceleration time from 50 to $100 \mathrm{keV}$ to be no shorter than about $35 \mathrm{~ms}$. This finite acceleration time must be allowed for in the interpretation of fast timescale features. For example, Aschwanden et al. (1995) attribute a delay between the $25-30$ and $50-100 \mathrm{keV}$ hard X-ray emission solely to energy-dependent propagation effects and thereby infer the height of the acceleration region above the interaction region (presumably the loop footpoints). Since we do not consider trapping in this paper, we (like Aschwanden et al.) have to make an assumption regarding release. However, we do take into account an energy dependence of the acceleration time. Assuming that electrons of 50 and $100 \mathrm{keV}$ are released instantaneously upon reaching these energies, that the time difference between releases is 35 $\mathrm{ms}$, and that the higher energy electrons reach the interaction region some $17 \mathrm{~ms}$ earlier, we see that the distance between acceleration and interaction regions is $\approx 2.6 \times 10^{9}$ $\mathrm{cm}$. This is larger than Aschwanden et al.'s estimate of $7.3 \times 10^{8} \mathrm{~cm}$, and a longer acceleration time increases the distance even more. Admittedly, this is a rough calculation, but it does illustrate the potential importance of a realistic acceleration time on such an analysis. 
For Kraichnan cascading, the injection wavelength needs to be smaller than in the Kolmogorov case, due to the longer cascade time and thus the greater importance of wave convective losses. This is why a $\tilde{\lambda}_{i}$ of 160 was used in the Kraichnan simulation in $\S 3$. Namely, we need $\tilde{k}_{i}^{1 / 2} \tilde{W}_{0}>1.2 \times 10^{-5}$ for an extended injection and $\tilde{k}_{i} \tilde{U}_{T i}>1.2 \times 10^{-5}$ for an impulsive injection. Using the above values of $\tilde{Q}$ and $\tilde{U}_{T i}$, a $\lambda_{i}$ smaller than $\approx 2 \times 10^{6} \mathrm{~cm}$ would satisfy either constraint. This upper limit could be increased through the generation of more turbulence, but $120 \mathrm{ergs} \mathrm{cm}^{-3}$ would be needed for a limit of $2 \times 10^{7} \mathrm{~cm}$ to be attained. Hence, relatively low wave levels require excitation at smaller scales than in the Kolmogorov case. We did not show results in $\S 3$ for an impulsive injection of turbulence, but, as with the Kolmogorov case, the essential electron results are the same as an extended injection. Keeping the turbulence to a minimum, then, we see that 12 ergs $\mathrm{cm}^{-3}$ of waves injected over any scale smaller than $\approx 2 \times 10^{6} \mathrm{~cm}$ and over any time interval smaller than $\approx 600$ ms will lead to an $F(>20 \mathrm{keV})$ of more than $5 \times 10^{7} \mathrm{~cm}^{-3}$ $\mathrm{s}^{-1}$ for $\approx 320 \mathrm{~ms}$ and an $N(>20 \mathrm{keV})$ of $\approx 4 \times 10^{7} \mathrm{~cm}^{-3}$. We thus conclude that this other cascading scenario is also consistent with observations.

As with wave convective losses, there is one other aspect of this model that needs to be mentioned but which is not taken into account in the simulations. This is electron Landau damping of relatively high-frequency fast mode waves. Landau damping and transit-time damping are similar insofar as they both involve the $l=0$ resonance, but they differ in regards to what the particle actually resonates with. In the case of transit-time damping, the interaction is between the parallel motion of the particle and the parallel component of the wave magnetic field, whereas for Landau damping the interaction is between the parallel motion of the particle and the parallel component of the wave electric field. In the MHD limit, the fast mode wave polarization is as discussed in $\S 1$ or the Appendix, and Landau damping is negligible since the wave parallel electric field is small. However, as the frequency increases beyond this regime, the transverse electric field becomes right-hand circularly polarized and a nonnegligible parallel electric field develops.

The change in the transverse electric field will decrease the efficiency of transit-time damping since half of the available electric field energy will be in a component (the $E_{x}$ component according to the convention in the Appendix) that does not produce a parallel magnetic field. While decreasing the strength of the $l=0$ interaction, this transverse field will increase the efficiency of the $l=+1$ gyroresonance, which can be achieved by relativistic electrons. At any rate, we do not expect this change to significantly affect our results for hard X-ray-producing electrons, since there will still be significant energy available for transit-time acceleration.

The parallel electric field will also decrease the efficiency of transit-time damping by increasing the efficiency of Landau damping, which transfers wave energy to electrons near the thermal speed and leads to heating. We now estimate the effect of Landau damping. From Stix (1992, p. 288), we can calculate the ratio of wave power absorption due to transit-time damping to that due to Landau damping. We find that

$$
\frac{P_{T}}{P_{L}}=\left(\frac{\Omega_{\mathrm{H}} \lambda_{e}}{c}\right)^{2} \beta_{e}\left(\frac{c k_{\|}}{\Omega_{\mathrm{H}}}\right)^{2}\left(\frac{c k_{\perp}}{\Omega_{\mathrm{H}}}\right)^{2}\left(\frac{\Omega_{\mathrm{H}}}{\omega}\right)^{2}\left(\frac{E_{y}}{E_{\|}}\right)^{2},
$$

where $\lambda_{e}$ is the electron Debye length; $\beta_{e}=n_{\mathrm{H}} k_{B} T / U_{B}$ is the electron plasma beta; $E_{y}$ and $E_{\|}$are the $y$ - and $z$ components of the wave electric field, respectively (assuming the wave propagates in the $x-z$ plane as in the Appendix); and $k_{\|}$and $k_{\perp}$ are the $z$ and $x$ components of the wavevector, respectively. This is a general relation and is valid unless $c k / \Omega_{\mathrm{H}}$ exceeds $\sim 10^{4}$. Using the cold plasma dispersion relation and the physical parameters of $\S 3$, and taking the propagation angle to be $45^{\circ}$, we find that $P_{T} / P_{L}$ goes as approximately $\omega^{-4}$ in the MHD regime, where it exceeds $\sim 10^{7}$. As the frequency increases, $P_{T} / P_{L}$ decreases but is greater than 1 for waves on the fast mode branch with $\omega / \Omega_{\mathrm{H}} \lesssim 0.7$ or $c k / \Omega_{\mathrm{H}} \lesssim 17$. Above this frequency, Landau damping is more rapid than transit-time damping.

In the simulations in $\S 3$, the maximum value attained by $c\langle k\rangle / \Omega_{\mathrm{H}}$ ranges from about 0.6 to 4 , which is in the range where transit-time damping dominates. However, it is clear that the spectral density extends to higher wavenumbers. For Kraichnan cascading, the spectral energy resides below $c k / \Omega_{\mathrm{H}} \approx 20$, and so Landau damping and electron heating are not of great importance here. For Kolmogorov cascading, though, waves can have $c k / \Omega_{\mathrm{H}}$ in excess of 100 , which is at the bottom of the whistler regime. In this case, bulk electron heating may be significant. While decreasing the efficiency of transit-time damping, heating is not an intrinsically undesired phenomenon, since there is observational evidence that it occurs (e.g., Lin \& Johns 1993) and since it will still permit efficient acceleration.

As the waves initially cascade to higher wavenumbers, Landau damping will dominate and the wave energy will go into heating rather than acceleration. However, for the wave injection rates we considered in $\S 3$, Landau damping will not lead to a cutoff in the spectral density at much lower wavenumbers and thus to an appreciable decrease of $\langle k\rangle$. Specifically, the rate at which wave energy density in an interval $\delta k$ about $k$ is absorbed due to Landau damping is approximately given by (from Stix 1992, p. 289)

$$
\begin{aligned}
\delta P_{L} \approx & 2 \sqrt{\pi}\left(\frac{\omega_{p e}}{\Omega_{\mathrm{H}}}\right)^{2}\left(\frac{c}{v_{t e}}\right)^{3}\left(\frac{E_{\|}}{E_{y}}\right)^{2}\left(\frac{\omega}{c k}\right)^{2} \\
& \times\left(\frac{\omega}{c k_{\|}}\right)^{2}\left(\frac{\Omega_{\mathrm{H}}}{c k_{\|}}\right) \\
& \times \exp \left[-\left(\frac{\omega}{k_{\|} v_{t e}}\right)^{2}\right] \Omega_{\mathrm{H}} W_{T}(k) \delta k,
\end{aligned}
$$

where $\omega_{p e}$ is the electron plasma frequency. In deriving this expression for $\delta P_{L}$, we have neglected the $x$-component of the wave electric field, so that it is uncertain to within a factor of $\approx 2$ above the MHD regime. The quantity $\delta P_{L} / \delta k$ rapidly increases with increasing frequency and attains a maximum value of $8 \times 10^{-13} U_{B} c$ at $\omega \approx 1.1 \Omega_{\mathrm{H}}$, above which it rapidly decreases. Below $1.1 \Omega_{\mathrm{H}}, \delta P_{L} / \delta k$ increases due to the $E_{\|} / E_{\mathrm{y}}$ factor (which becomes larger as $\Omega_{\mathrm{H}}$ is approached), while above this frequency $\delta P_{L} / \delta k$ decreases due to the larger wave parallel phase speed (which becomes much larger than the electron thermal speed). Below $1.1 \Omega_{\mathrm{H}}$ then, an upper limit on the integral power absorption $P_{L}=$ $\int_{0}^{k} d k \delta P_{L} / \delta k$ is obtained by letting $\delta k=k$ in equation (4.2). Above $1.1 \Omega_{\mathrm{H}}$, any further contribution is small. If $P_{L}=Q$, where $Q$ is the volumetric rate of energy transport to higher wavenumbers (see discussion in $\S 3$ ), then the spectral density will turn over due to Landau damping at that wavenumber. However, taking a typical wave propagation angle 
of $45^{\circ}$ and employing the cold plasma dispersion relation, we find that for a wave energy density injection rate of $Q=4 \times 10^{-10} U_{B} \Omega_{\mathrm{H}}$, the Landau absorption rate $P_{L}$ reaches a maximum of $\approx 1.9 \times 10^{-11} U_{H} \Omega_{H}$ at $\omega=1.1 \Omega_{\mathrm{H}}$, above which it remains about constant. As $P_{L}$ never exceeds $Q$, some wave energy will be free to cascade to high wavenumbers as before.

In the Kolmogorov phenomenology then, some wave energy will be dissipated on heating during the initial cascade to high wavenumbers, but since the volumetric cascade rate exceeds the heating rate, $\langle k\rangle$ will not be significantly affected and the acceleration rate should remain high. Note also that (1) heating decreases the Coulomb energy loss rate for electrons above the threshold velocity, thus facilitating the acceleration of these electrons and (2) that heating will increase the total number above $v_{\mathrm{A}}$. Hence, while heating will be important in some cases (namely, lower values of $Q$ ), we do not expect it to affect the viability of transit-time acceleration.

The last item we wish to point out concerning transittime electron acceleration is its relationship to ion acceleration. Specifically, fast mode waves offer an opportunity to unify ion and electron acceleration within the context of MHD turbulence. Such turbulence, in general, consists of shear Alfvén, fast mode, and slow mode waves, and the effect of the fast mode is well discussed above. The slow mode is heavily Landau damped, which may prevent its excitation. If it is generated, it will heat the electrons and further increase the efficiency of transit-time acceleration by boosting the number of electrons above the threshold speed. Miller \& Roberts (1995) and Miller \& Reames (1995; see also Miller \& Dermer 1995) have shown that cascading shear Alfvén waves preferentially accelerate ions from the thermal distribution to relativistic energies and can account for solar flare gamma-ray line emission, as well as the heavy ion abundances observed in the interplanetary particles. Therefore, we can now envisage low-frequency MHD waves being excited during the flare energy release and subsequently cascading to higher frequencies where they are damped - the fast mode waves on the electrons and the shear Alfvén waves on the ions. In this way, each of the two MHD modes that are not heavily Landau damped will accelerate a separate particle species.

The ion that is not included in this cascading theory is ${ }^{3} \mathrm{He}$, which almost certainly is at least preaccelerated by waves excited around its cyclotron frequency. It has been previously shown that bump-on-tail electron distributions are unstable to electromagnetic hydrogen cyclotron waves in a solar flare plasma, and that these waves can efficiently accelerate ${ }^{3} \mathrm{He}$ and lead to large ${ }^{3} \mathrm{He} /{ }^{4} \mathrm{He}$ abundance enhancements (Temerin \& Roth 1992; Miller \& Viñas 1993). However, the source of the necessary electron distribution was unknown. In $\S 3$ we showed that bump-on-tail distributions in energy space can result from transit-time damping, with the bump appearing at an energy of $\approx 5 \mathrm{keV}$ (as required in the ion studies). While this is not equivalent to a velocity-space anisotropy, such a bump may appear in parallel velocity space as well. A more detailed treatment that includes escape, Landau damping, and the selfexcitation of lower hybrid waves is necessary to verify this.

\section{SUMMARY}

We considered the cascading of fast mode waves from low to high frequencies in a solar flare plasma, and the resulting stochastic acceleration of electrons out of the thermal distribution and up to ultrarelativistic energies by transit-time damping. Our specific results are as follows:

1. With a Kolmogorov phenomenology, the generation of $12 \mathrm{ergs} \mathrm{cm}^{-3}$ of fast mode wave turbulence on any scale less than $\approx 10^{8} \mathrm{~cm}$ and over any time interval less than $\approx 600$ ms will yield a specific acceleration rate above $20 \mathrm{keV}$ of more than $5 \times 10^{7} \mathrm{~cm}^{-3} \mathrm{~s}^{-1}$ for $240-320 \mathrm{~ms}$ and a total number density above $20 \mathrm{keV}$ of $4-6 \times 10^{7} \mathrm{~cm}^{-3}$.

2. With a Kraichnan phenomenology, the generation of

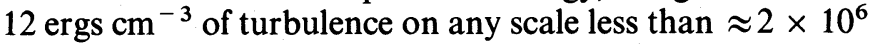
$\mathrm{cm}$ and over any time interval less than $\approx 600 \mathrm{~ms}$ will yield the same specific acceleration rate and total number density.

3. Assuming an acceleration region volume of $10^{27} \mathrm{~cm}^{3}$, this specific acceleration rate and total electron density above $20 \mathrm{keV}$ can account for the energy-integrated hard X-ray flux observed during a subsecond spike or energy release fragment in impulsive solar flares. The acceleration region size and the total wave energy density determine the upper limit on the injection wavelength through wave convective escape. A larger volume would increase this upper limit as would a higher injected turbulence energy density.

4. The electron spectra resulting from transit-time damping have the potential to be very hard and can attain an $E^{-1.2}$ form between 20 and $100 \mathrm{keV}$. Such spectra are harder than that resulting from diffusive shock acceleration, but taking into account escape may affect this result. They are also harder than those implied by hard X-ray observations (which typically indicate an $E^{-4.5}$ spectrum). Again, escape is expected to soften the spectra, as is the inclusion of Landau damping (since Landau damping will increase the number of electrons above threshold and thus decrease the average energy per electron for a fixed amount of injected turbulence).

5. Electrons are accelerated to ultrarelativistic $(\gamma \gtrsim 10)$ energies, and so an additional mechanism operable at high energies is not needed (as is the case with large-scale electric fields).

6. Electron heating will accompany acceleration in the Kolmogorov phenomenology but will not be significant in the Kraichnan phenomenology. The precise amount of heating in the first instance, and the detailed effect on the electron distribution, can only be determined with a more comprehensive simulation but should not destroy the viability of transit-time acceleration or lead to larger energy densities than those given above.

7. This mechanism establishes an attractive unified ion/ electron acceleration model. During the primary flare energy release, MHD turbulence (consisting of shear Alfvén and fast mode waves) is generated at large scales and subsequently cascades to higher frequencies through an inertial range. At large wavenumbers a dissipation range forms, where damping of the Alfvén waves is due to the ambient ions and damping of the fast mode waves is due to the electrons. In this way, MHD turbulence is capable of accounting for the near simultaneous electron and ion acceleration that is observed from impulsive solar flares.

Future work on this model will focus on the anomalous Doppler resonance instability and its role in isotropizing the electrons and return currents, and accounting for longer timescale hard X-ray emission, electron heating, and the fitting of observed hard X-ray spectra. 
We thank P. Cargill for important discussions, and he and G. Emslie for critical readings of the manuscript. J. A. M. was supported by the NASA Cosmic and Heliospheric Physics Program, and by the NSF Solar Terrestrial
Physics Program; T. N. L. was supported by a NASA JOint VEnture (JOVE) grant; and R. L. M. was supported by the NASA Solar Physics Program.

\section{APPENDIX}

TRANSIT-TIME DAMPING MOMENTUM DIFFUSION COEFFICIENT

The magnetic vector potential $\boldsymbol{A}_{w}$ for a spectrum of $N$ monochromatic low-frequency fast mode waves propagating in the $x-z$ plane is given by

$$
A_{w}=\sum_{i=1}^{N} \frac{c E_{i}}{\omega_{i}} \hat{y} \cos \phi_{i}
$$

where $\phi_{i}=k_{\perp i} x+k_{\| i} z-\omega_{i} t+\phi_{0 i}$. The quantities $\boldsymbol{k}_{i}=k_{\perp i} \hat{x}+k_{\| i} \hat{z}, \omega_{i}, E_{i}$, and $\phi_{0 i}$ are the wavevector, frequency, electric field amplitude, and constant phase offset of wave $i$, respectively. Each wave is completely electromagnetic, and the scalar potential is zero. The vector potential $\boldsymbol{A}_{0}$ of the background magnetic field is $-y B_{0} x$, so that the relativistically correct Hamiltonian for an electron of charge $-e$ and mass $m$ becomes

$$
H=m c^{2}\left[\left(\frac{P_{x}}{m c}-\frac{\Omega}{c} y\right)^{2}+\left(\frac{P_{y}}{m c}+\sum_{i=1}^{N} \epsilon_{i} \cos \phi_{i}\right)^{2}+\left(\frac{P_{\|}}{m c}\right)^{2}+1\right]^{1 / 2},
$$

where the normalized electric field $\epsilon_{i}=e E_{i} / m c \omega_{i}$ and the classical gyrofrequency $\Omega=e B_{0} / m c$. Here $P_{x}, P_{y}$, and $P_{\|}$are the components of the canonical momentum $\boldsymbol{P}=\boldsymbol{p}-e\left(\boldsymbol{A}_{w}+\boldsymbol{A}_{0}\right) / c$, where $\boldsymbol{p}=p_{x} \hat{\boldsymbol{x}}+p_{y} \hat{\boldsymbol{y}}+p_{\|} \hat{z}$ is the ordinary mechanical momentum. However, since the parallel component of $A_{0}+A_{w}$ is zero, we have that $P_{\|}=p_{\|}$.

Since the waves are small amplitude, we can resolve the electron trajectory into slow guiding-center drift and fast gyration about the guiding center. We thus transform to guiding-center action-angle variables using the generating function

$$
F_{1}\left(x, y, y_{g}, \theta\right)=m \Omega\left[-\frac{1}{2}\left(y-y_{g}\right)^{2} \tan \theta+x y_{g}\right],
$$

where $\left(x, P_{x}\right),\left(y, P_{y}\right),\left(y_{g}, P_{g y}\right)$, and $(\theta, J)$ are conjugate pairs. The pair $\left(z, P_{\|}\right)$is unaffected by the transformation. Here, $y_{g}$ and $x_{g}=P_{g y} / m \Omega$ are the $y$ - and $x$-coordinates of the guiding-center frame origin. We define two ancillary variables, $P_{\perp}=$ $(2 \mathrm{Jm} \Omega)^{1 / 2}$ and $\rho=P_{\perp} / m \Omega$, which, in the limit of $\epsilon_{i} \rightarrow 0$, become the mechanical perpendicular momentum and gyroradius in the guiding-center frame. Transforming $H$, expanding the result to first order in $\epsilon_{i}$, and using the Bessel function expansion of the cosine, we obtain

$$
H=m c^{2} \gamma+\frac{m c^{2}}{\gamma}\left(\frac{P_{\perp}}{m c}\right) \sum_{i=1}^{N} \epsilon_{i} \sum_{l=-\infty}^{+\infty} J_{l}^{\prime}\left(k_{\perp i} \rho\right) \sin \left(k_{\| i} z-\omega_{i} t+l \theta+\delta_{i}\right)
$$

where $\delta_{i}=k_{\perp i} x_{g}+\phi_{0 i}$ is another constant, $J_{l}^{\prime}$ is the derivative of the Bessel function, and $\gamma^{2}=\left(P_{\perp} / m c\right)^{2}+\left(P_{\|} / m c\right)^{2}+1$. Resonance between the electron and wave $i$ will occur when $k_{\| i} \dot{z}-\omega_{i}+l \dot{\theta}=0$ (the dot denotes time differentiation). Using the Hamilton equations of motion and approximating $H$ by the first term in equation (A4), the resonance condition becomes $\omega_{i}-k_{\| i} p_{\|} / m \gamma-l \Omega / \gamma=0$. Since terms of order $\epsilon_{i}$ have already been neglected in deriving this relation, it is consistent to set $\gamma$ equal to the Lorentz factor, in which case this equation becomes the usual resonance condition.

The only resonance that can be achieved by nonrelativistic electrons is $l=0$. The resonance condition for this harmonic number becomes $v_{\|}=v_{\phi \|}$, where $v_{\phi \|}=\omega_{i} / k_{\| i}$ is the parallel phase speed of the wave and $v_{\|}$is the parallel electron speed. Using the fast mode dispersion relation, we can rewrite this condition as $v_{\|}=v_{\mathrm{A}} / \eta$, where $\eta=\boldsymbol{k} \cdot \boldsymbol{B}_{0} /|\boldsymbol{k}| \boldsymbol{B}_{0}$ is the wave propagation angle cosine. Hence, $\left|v_{\|}\right|$must be greater than $v_{\mathrm{A}}$ before interactions with fast mode waves can occur, so that $v_{\mathrm{A}}$ is the acceleration threshold speed.

Using the Hamilton equations $\dot{P}_{\|}=-\partial H / \partial z$ and $\dot{J}=-\partial H / \partial \theta$, the above definition of $P_{\perp}$, and then approximating $P_{\perp}$ by the mechanical momentum $p_{\perp}$, we find that

$$
\dot{p}_{\perp}=0 \text {, }
$$

and

$$
\dot{p}_{\|}=-\frac{m c^{2}}{\gamma}\left(\frac{p_{\perp}}{m c}\right) \sum_{i=1}^{N} \epsilon_{i} k_{\| i} J_{0}^{\prime}\left(k_{\perp i} \rho\right) \cos \left(k_{\| i} z-\omega_{i} t+\delta_{i}\right),
$$

where $\gamma$ is now the Lorentz factor. Hence, the $l=0$ resonance leads only to a change in the parallel energy. The diffusion coefficient $D_{\|||}$is given by (e.g., Schlickeiser \& Achatz 1993)

$$
D_{\|\|} \equiv \frac{\left\langle\Delta p_{\|} \Delta p_{\|}\right\rangle}{2 \Delta t}=\int_{0}^{\infty} d \tau\left\langle\dot{p}_{\|}(t) \dot{p}_{\|}(t+\tau)\right\rangle,
$$


where the integrand is the correlation function and the angle brackets denote an average over initial conditions. In this case,

$$
\langle\rangle=\frac{1}{2 \pi} \int_{0}^{2 \pi} d \theta_{0} \frac{1}{2 a} \int_{-a}^{+a} d z_{0},
$$

where $\theta_{0}$ and $z_{0}$ are the initial gyrophase angle and $z$-position, respectively, and the particle was assumed to have been initially confined to interval $[-a,+a]$. Using the unperturbed orbits $\theta(t)=\theta_{0}-\Omega t / \gamma$ and $z(t)=z_{0}+v_{\|} t$ to evaluate the phase terms, we find after some algebra that

$$
D_{\|\|}=\frac{\pi}{2}\left(\frac{p_{\perp}}{\gamma}\right)^{2} \Omega^{2} \sum_{i=1}^{N} \eta_{i}^{2}\left(\frac{B_{i}}{B_{0}}\right)^{2} J_{1}^{2}\left(k_{\perp i} \rho\right) \delta\left(\omega_{i}-k_{\| i} v_{\|}\right),
$$

where $B_{i}$ and $n_{i}$ are the magnetic field amplitude and propagation angle cosine of wave $i$, respectively. We use the wave magnetic field because $B_{i}=c E_{i} / v_{\mathrm{A}}$ and is therefore much greater than $E_{i}$ for solar values of $v_{\mathrm{A}}$. Consequently, essentially all of the wave vacuum field energy lies in $B_{i}$. Since $k_{\perp i} \rho \ll 1$ for the electron energies and waves under consideration, the Bessel function can be accurately approximated as $k_{\perp i} \rho / 2$.

For a discrete spectrum of waves, equation (A8) is not useful in its present form, and some type of resonance broadening must be introduced (e.g., Karmimabadi \& Menyuk 1991). However, we are interested in a continuous wave spectrum, such as that resulting from cascading. We generalize to a continuous spectrum of waves by letting $\sum_{B} B_{i}^{2} / B_{0}^{2}=\left(2 / U_{B}\right)$ $\sum B_{i}^{2} / 16 \pi \rightarrow\left(2 / U_{B}\right) \int d \eta d k W_{B}(\eta, k)$, where $U_{B}=B_{0}^{2} / 8 \pi$ is the ambient magnetic field energy density and $W_{B}$ is the magnetic energy spectral density. Writing $\delta\left(\omega-k_{\|} v_{\|}\right)$as $k^{-1} \delta\left(v_{\mathrm{A}}-\eta v_{\|}\right)$, performing the integration over $\eta$, and assuming isotropic turbulence [so that $\left.W_{B}(\eta, k)=W_{B}(k) / 2\right]$, we find that

$$
D_{\|\|}=(m c)^{2} \Omega_{\mathrm{H}} \frac{\pi}{8}\left(\frac{p_{\perp}}{m c}\right)^{4} \frac{1}{\gamma^{2}}\left(\frac{c}{\left|v_{\|}\right|}\right)^{3}\left(1-\frac{v_{\mathrm{A}}^{2}}{v_{\|}^{2}}\right)\left(\frac{v_{\mathrm{A}}}{c}\right)^{2} \frac{1}{U_{B}} \int_{0}^{\infty} d k\left(\frac{c k}{\Omega_{\mathrm{H}}}\right) W_{B}(k)
$$

for $\left|v_{\|}\right|>v_{\mathrm{A}}$. If $\left|v_{\|}\right|<v_{\mathrm{A}}, D_{\|\|}=0$. Here, $\Omega_{\mathrm{H}}$ is the hydrogen cyclotron frequency, and we have written $D_{\|\|}$in this manner in order to facilitate the use of dimensionless variables and to readily reveal the dimensional correctness of the expression. We normalize time to $\Omega_{\mathrm{H}}^{-1}$ instead of $\Omega^{-1}$ since (1) the waves are in the MHD regime and dimensionless wave numbers will be of order unity, and (2) the acceleration timescales are closer to $\Omega_{\mathrm{H}}^{-1}$ for the levels of turbulence that we employ. Casting this equation into an even more useful form, we have

$$
D_{\|\|}=(m c)^{2} \Omega_{\mathrm{H}} \frac{\pi}{16}\left(\frac{p_{\perp}}{m c}\right)^{4} \frac{1}{\gamma^{2}}\left(\frac{c}{\left|v_{\|}\right|}\right)^{3}\left(1-\frac{v_{\mathrm{A}}^{2}}{v_{\|}^{2}}\right)\left(\frac{v_{\mathrm{A}}}{c}\right)^{2} \frac{c\langle k\rangle}{\Omega_{\mathrm{H}}} \frac{U_{T}}{U_{B}},
$$

where $U_{T}=2 \int_{0}^{\infty} d k W_{B}(k)$ is the total (kinetic plus vacuum field) energy density of the fast mode waves and $c\langle k\rangle / \Omega_{\mathrm{H}}$ is the mean dimensionless wavenumber of the waves. This result is completely general and is independent of the angular distribution of the particles. Note that $D_{\|\|} \rightarrow 0$ as $p_{\perp} \rightarrow 0$, as expected, since mirroring cannot occur for particles of zero pitch angle.

If isotropizing scattering is introduced, it is more appropriate to use the $D_{p p}$ diffusion coefficient in spherical momentum coordinates. Since $p^{2}=p_{\|}^{2}+p_{\perp}^{2}$, we have from equations (A5a) and (A6) that $D_{p p}=\mu^{2} D_{\|\|}$, where $\mu$ is the cosine of the particle pitch angle. The $D_{p p}$ coefficient can now be averaged over $\mu$ to obtain the isotropic momentum diffusion coefficient $D(p)$.

Achterberg, A. 1979, A\&A, 76, 276

$$
\text { 1981, A\&A, 97, } 259
$$

An, Z. G., Liu, C. S., Lee, Y. C., \& Boyd, D. A. 1982, Phys. Fluids, 25, 997

Aschwanden, M. J., Schwartz, R. A., \& Alt, D. M. 1995, ApJ, 447, 923

Benka, S. G., \& Holman, G. D. 1994, ApJ, 435, 469

Benz, A. O. 1985, Sol. Phys., 104, 99

Brown, J. C. 1971, Sol. Phys., 18, 489

Chiueh, T., \& Zweibel, E. G. 1987, ApJ, 317, 900

Davis, L. 1956, Phys. Rev., 101, 351

de la Beaujardière, J.-F., \& Zweibel, E. G. 1989, ApJ, 336, 1059

Eichler, D. 1979, ApJ, 229, 409

Emslie, A. G. 1980, ApJ, 235, 1055

Emsie, 1983, ApJ, 271, 367

Emslie, A. G., \& Hénoux, J. C. 1995, ApJ, 446, 371

Fermi, E. 1949, Phys. Rev., 75, 1169

Fisk, L. A. 1976, J. Geophys. Res., 81, 4633

Forman, M. A., Ramaty, R., \& Zweibel, E. G. 1986, in Physics of the Sun Vol. 2, ed. P. A. Sturrock (Dordrecht: Reidel), 249

Gisler, G. 1992, in Particle Acceleration in Cosmic Plasmas, ed. G. P. Zank \& T. K. Gaisser (New York: AIP), 229

Gisler, G., \& Lemons, D. 1990, J. Geophys. Res., 95, 14925

Güdel, M., Aschwanden, M. J., \& Benz, A. O. 1991, A\&A, 251, 285

Hoyng, P., Brown, J. C., \& van Beek, H. F. 1976, Sol. Phys., 48, 197

Huba, J. D. 1994, NRL Plasma Formulary (Washington, DC: Naval Research Laboratory), NRL/PU/6790-94-265

Karimabadi, H., Krauss-Varban, D., \& Terasawa, T. 1992, J. Geophys. Res., 97,13853

Karimabadi, H., \& Menyuk, C. R. 1991, J. Geophys. Res., 96, 9669

\section{REFERENCES}

Karimabadi, H., Omidi, N., \& Gary, S. P. 1994, in Solar System Plasmas in Space and Time, Geophys. Monograph 84, ed. J. Burch \& J. H. Waite (Washington, DC: AGU), 221

Kiplinger, A. L., Dennis, B. R., Frost, K. J., \& Orwig, L. E. 1984, ApJ, 287, L105

LaRosa, T. N., \& Emslie, A. G. 1989, Sol. Phys., 120, 343

LaRosa, T. N., \& Moore, R. L. 1993, ApJ, 418, 912

LaRosa, T. N., Moore, R. L., \& Shore, S. N. 1994, ApJ, 425, 856, 1994

Lin, R. P., \& Johns, C. M. 1993, ApJ, 417, L53

Liu, C. S., \& Mok, Y. 1977, Phys. Rev. Lett., 38, 162

MacDonald, W. M., Rosenbluth, M. N., \& Chuck, W. 1957, Phys. Rev., 107,350

Machado, M. E., Ong, K. K., Emslie, A. G., Fishman, G. J., Meegan, C., Wilson, R., \& Paciesas, W. S. 1993, Adv. Space Res., 13(9), 175

Marsch, E. 1991, in Physics of the Inner Heliosphere II, ed. R. Schwenn \& E. Marsch (Berlin: Springer)

Miller, J. A. 1991, ApJ, 376, 342

Miller, J. A., \& Dermer, C. D. 1995, A\&A, 298, L13

Miller, J. A., \& Reames, D. V. 1995, ApJ, in preparation

Miller, J. A., \& Roberts, D. A. 1995, ApJ, 452, 912

Miller, J. A., \& Viñas, A. F. 1993, ApJ, 412, 386

Moghaddam-Taaheri, E., Vlahos, L., Rowland, H. L., \& Papadopoulos, K. 1985, Phys. Fluids, 28, 3356

Press, W. H., Flannery, B. P., Teukolsky, S. A., \& Vetterling, W. T. 1986, Numerical Recipes: The Art of Scientific Computing (Cambridge: Cambridge Univ. Press)

Ramaty, R. 1979, in Particle Acceleration Mechanisms in Astrophysics, ed J. Arons, C. Max, \& C. McKee (New York: AIP), 135 


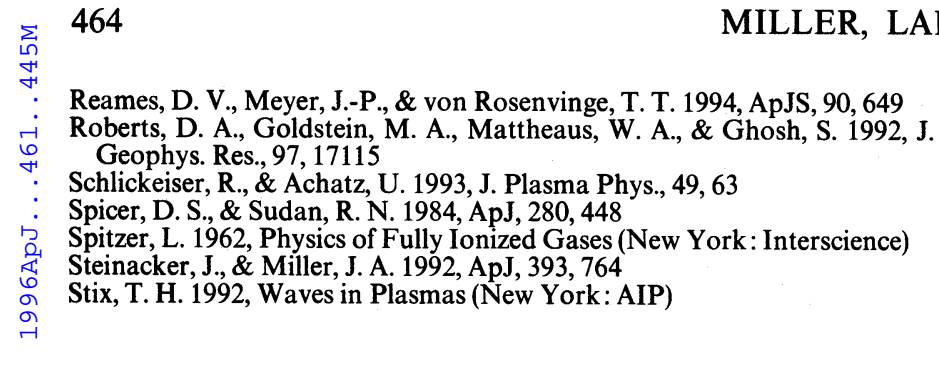

IT. Reames, D. V., Meyer, J.-P., \& von Rosenvinge, T. T. 1994, ApJS, 90, 649

'1 Roberts, D. A., Goldstein, M. A., Mattheaus, W. A., \& Ghosh, S. 1992, J.

Geophys. Res., 97, 17115

Schlickeiser, R., \& Achatz, U. 1993, J. Plasma Phys., 49, 63

I Spicer, D. S., \& Sudan, R. N. 1984, ApJ, 280, 448

I'I Spitzer, L. 1962, Physics of Fully Ionized Gases (New York: Interscience)

, Steinacker, J., \& Miller, J. A. 1992, ApJ, 393, 764

'. Stix, T. H. 1992, Waves in Plasmas (New York: AIP)

Iㄴ.

MILLER, LAROSA, \& MOORE

Swanson, D. G. 1989, Plasma Waves (New York: Academic)

Temerin, M., \& Roth, I. 1992, ApJ, 391, L105

Tsytovich, V. N. 1966, Soviet Phys.-Uspekhi, 9(3), 370

van den Oord, G. H. J. 1990, A\&A, 234, 496

Zhou, Y., \& Matthaeus, W. H. 1990, J. Geophys. Res., 95, 14881

Zweibel, E. G., \& de la Beaujardière, J.-F. 1990, Geophys. Res. Lett., 17, 205 